\title{
The Matrix Product Approach to Quantum Spin Ladders
}

\author{
J.M. Román ${ }^{1}$, G. Sierra ${ }^{2}$, J. Dukelsky ${ }^{3}$, M.A. Martín-Delgado ${ }^{4}$, \\ ${ }^{1}$ Departament d'E.C.M., Universitat de Barcelona, Barcelona, Spain. \\ ${ }^{2}$ Instituto de Matemáticas y Física Fundamental, C.S.I.C., Madrid, Spain. \\ ${ }^{3}$ Instituto de Estructura de la Materia, C.S.I.C.,Madrid, Spain. \\ ${ }^{4}$ Departamento de Física Teórica I, Universidad Complutense. Madrid, Spain.
}

\begin{abstract}
We present a manifestly rotational invariant formulation of the matrix product method valid for spin chains and ladders. We apply it to 2 legged spin ladders with spins $1 / 2,1$ and $3 / 2$ and different magnetic structures labelled by the exchange coupling constants, which can be ferromagnetic or antiferromagnetic along the legs and the rungs of the ladder We compute ground state energy densities, correlation lengths and string order parameters. We present numerical evidence of the duality properties of the 3 different non ferromagnetic spin $1 / 2$ ladders. We show that the long range topological order characteristic of isolated spin 1 chains is broken by the interchain coupling. The string order correlation function decays exponentially with a finite correlation length that we compute. A physical picture of the spin 1 ladder is given in terms of a collection of resonating spin 1 chains. Finally for ladders with spin equal or greater than $3 / 2$ we define a class of AKLT states whose matrix product coefficients are given by 9 -j symbols.
\end{abstract}

\section{I) INTRODUCTION}

The matrix product method (MPM) is a variational approach appropiate to study the ground state and excitations of a variety of one-dimensional lattice systems in Condensed Matter and Statistical Mechanics. The theoretical and experimental interest of these systems has grown spectacularly in the last years, due to the discovery of interesting and unexpected physical properties in spin chains and ladders.

The basic idea behind the MPM is the construction of the ground state and excitations of $1 \mathrm{D}$ or quasi $1 \mathrm{D}$ systems in a recursive way, by relating the states of the system with length $N$ to that of length $N-1$. This simple idea has appeared in the past in different places. First of all, in the Wilson's real space renormalization group the $1 \mathrm{D}$-lattice is built up by the addition of a single site at every RG step [1]. This procedure is also used in the density matrix renormalization group method (DMRG) of White [2]. Other source of the MPM is the well known AKLT state of the spin 1 chain [3]. This is a simple but non trivial example of a matrix product state, which has motivated various generalizations as the ones of Klumper et al [4, Ostlund and Rommer [5], etc. We shall follow in this paper the formulation of the MPM due to the latter authors, which is based on the analysis of the fixed point structure of the DMRG ground state in the thermodynamic limit [5]. A closely related approach is that of Fannes et al [6]. The MPM offers an alternative formulation of the DMRG method in the regime where the latter reaches a fixed point after many RG iterations [7.

Whereas the DMRG is a purely numerical method, the MPM offers the possibility of an analytical approach to elucidate the actual structure of the ground state (GS) and excitations. The MPM is a standard variational method which determines the variational parameters by minimizing the GS energy. Minimization problems are in general harder than diagonalization ones. In this respect the MPM is so far less performant than the DMRG. However we believe that the analytical insights gained with the MPM could be used to boost the numerical precision and applications of both the MPM and the DMRG.

In this paper we apply the MPM to the 2-leg spin ladder. Spin ladders with diagonal couplings have been studied with the MPM of Klumper et al in ref. [8]. Spin ladders were first studied as theoretical labs to test ideas concerning the crossover from $1 \mathrm{D}$ to $2 \mathrm{D}$, with the surprising result that this crossover is far from being smooth: the even and odd ladders display quite different properties converging only when the number of legs goes to infinity (for an introduction to the subject see [9]). Even spin ladders are spin liquids with a finite spin gap and finite spin correlation length, while odd spin ladders belong to the same universality class than the spin $1 / 2$ antiferromagnetic Heisenberg chain, which has no gap and the correlations decay algebraically. Another reason to study ladder systems is that materials actually exists with that structure and hence the theoretical predictions can in principle be compared with experimental data concerning the spectrum, susceptibility, etc.

We study in this paper five different spin ladders characterized by their local spin $S=1 / 2,1$ and $3 / 2$ and the signs of the exchange of coupling constants along the legs $J_{\|}$and the rungs $J_{\perp}$. 
In the case of the spin $1 / 2$ ladders we discuss the following topics: i) the RVB picture of the antiferromagnetic ladder, ii) the equivalence between the ladder state and the Haldane state of the spin 1 chain, and iii) the duality properties relating the different magnetic structures.

In the case of the spin 1 ladder we show that the long range topological order characteristic of isolated spin 1 chains disappears and the string correlator decays exponentially with a finite correlation length.

The study of the spin 3/2 ladder motivates the definition of an AKLT state characterized in terms of 9-j symbols.

We compute GS energy densities, spin correlation lengths and string order parameters and compare our results with those existing in the current literature.

The organization of the paper is as follows. In section II we review the MPM. In section III we particularize the MPM to systems which are rotational invariant, where the use of group theory leads to a simplification of the formalism. In section IV we present our numerical results concerning five different spin ladders. In section $\mathrm{V}$ we summarize our results and present some prospects of our work. There are 3 appendices which contain technical details or proofs of results presented in the main body of the paper.

\section{II) REVIEW OF THE MATRIX PRODUCT METHOD}

Some of the results presented in this section are known and can be found in references [5.6. W. We also present a full account of the formulas and derivations used in reference [7] where the MPM was compared with the DMRG method in the case of the antiferromagnetic spin 1 chain.

Let us consider a spin chain or a ladder $B_{N}$ with open boundary conditions, where $N$ denotes the number of sites of a chain or the number of rungs of a ladder. To describe the low energy properties of $B_{N}$ one introduces a collection of $m$ states $\left\{|\alpha\rangle_{N}\right\}_{\alpha=1}^{m}$, which form an orthonormal basis, i.e. ${ }_{N}\left\langle\alpha \mid \alpha^{\prime}\right\rangle_{N}=\delta_{\alpha, \alpha^{\prime}}$. In the DMRG these states are the most probable ones to contribute to the GS of the superblock $B_{N-1} \bullet \bullet B_{N-1}^{R}$ of length $2 N$, formed by adding two sites (or rungs) $\bullet \bullet$, and a mirror image $B_{N-1}^{R}$ to the original lattice $B_{N-1}$. The basic assumption of the MPM is that the basis associated with $B_{N}$ and $B_{N-1}$ are related in a simple manner by the equation

$$
|\alpha\rangle_{N}=\sum_{\beta, s} A_{\alpha, \beta}[s]|s\rangle_{N} \otimes|\beta\rangle_{N-1} \quad, N \geq 2
$$

where $|s\rangle_{N}\left(s=1, \ldots, m^{*}\right)$ denotes a complete set of $m^{*}$ states associated to the $N^{\text {th }}$ site (resp. rung) added to the chain ( resp. ladder). Eq. (11) has to be supplemented with the initial data $|\beta\rangle_{1}$. The quantities $A_{\alpha, \beta}[s]$ are the variational parameters of the MPM, and their determination is the central problem one has to solve. This is done by the standard variational method. The important point about eq.(11) is that $A_{\alpha, \beta}[s]$ does not depend on $N$. Eq.(11) is motivated by the truncation method used in the DMRG where $A_{\alpha, \beta}[s]$ dependend on $N$, i.e. $A_{\alpha, \beta}^{(N)}[s]$. When $N$ is large enough one reaches a fixed point, i.e. $A_{\alpha, \beta}^{(N)}[s] \rightarrow A_{\alpha, \beta}[s]$. In this manner the thermodynamic limit of the DMRG leads to a translational invariant MPM state.

The condition that both $|\alpha\rangle_{N}$ and $|\beta\rangle_{N-1}$ form orthonormal basis imposes a normalization condition on $A_{\alpha, \beta}[s]$,

$$
\sum_{\beta, s} A_{\alpha, \beta}^{*}[s] A_{\alpha^{\prime}, \beta}[s]=\delta_{\alpha, \alpha^{\prime}}
$$

It is interesting to count how many variational parameters there are in (11). The quantities $A_{\alpha, \beta}[s]$ represent a total of $m^{2} m^{*}$ variables. We shall assume that all of them may be non vanishing. The normalization constraints (2) represent a total of $m+m(m-1) / 2$ constraints $(m$ coming from the diagonal terms $\alpha=\alpha^{\prime}$ and $m(m-1) / 2$ coming from the off-diagonal ones). On the other hand one can rotate the basis of states $\{|\alpha\rangle\}_{\alpha=1}^{m}$ by an element of the orthogonal group $O(m)$ reducing by $m(m-1) / 2$ the number of independent MPM variables. The total number of variational degrees of freedom, $N_{A}$, is then given by,

$$
N_{A}=m^{2} m^{*}-m-2 m(m-1) / 2=m^{2}\left(m^{*}-1\right)
$$

We show in appendix A that the set of $A_{\alpha, \beta}[s]$ belongs, to the grassmanian manifold,

$$
A \in \frac{O\left(m m^{*}\right)}{O(m) \otimes O\left(m\left(m^{*}-1\right)\right)}
$$

As an exercise one can check that the dimension of (4) coincides with $N_{A}$ given in (3).

In ref. [5] eq.(1) is used to generate an ansatz for the GS of periodic chains. In this paper we shall rather use this eq. to generate states with open boundary conditions, in the spirit of the DMRG. We shall show below that the set $|\alpha\rangle$ correspond to ground states with different boundary conditions. The use of open boundary conditions leads to a simplification of the MPM which is very close to the more abstract formalism proposed in [6].

\section{Correlators of local operators}

Let us use eq.(1) to compute the expectation values of local operators in a recursive way. We shall first consider a local operator $\mathcal{O}_{n}$ acting at the position $n=1, \ldots, N$ of the lattice. It is easy to get from (11) the expectation value, 


$$
\begin{gathered}
{ }_{N}\left\langle\alpha\left|\mathcal{O}_{n}\right| \alpha^{\prime}\right\rangle_{N} \\
= \begin{cases}\sum_{\beta \beta^{\prime}} T_{\alpha \alpha^{\prime}, \beta \beta^{\prime}}{ }^{N-1}\left\langle\beta\left|\mathcal{O}_{n}\right| \beta^{\prime}\right\rangle_{N-1} & \text { for } n<N \\
\sum_{\beta} \widehat{\mathcal{O}}_{\alpha \alpha^{\prime}, \beta \beta} & \text { for } n=N\end{cases}
\end{gathered}
$$

where

$$
\begin{gathered}
T_{\alpha \alpha^{\prime}, \beta \beta^{\prime}}=\sum_{s} A_{\alpha, \beta}^{*}[s] A_{\alpha^{\prime}, \beta^{\prime}}[s] \\
\widehat{\mathcal{O}}_{\alpha \alpha^{\prime}, \beta \beta^{\prime}}=\sum_{s s^{\prime}} A_{\alpha, \beta}^{*}[s] A_{\alpha^{\prime}, \beta^{\prime}}\left[s^{\prime}\right]\left\langle s|\mathcal{O}| s^{\prime}\right\rangle
\end{gathered}
$$

$T$ can be identified with $\widehat{1}$. Eqs.(5),6., (7) suggest to interpret the expectation value ${ }_{N}\left\langle\alpha\left|\mathcal{O}_{n}\right| \alpha^{\prime}\right\rangle_{N}$ as a vector labeled by the pair $\alpha \alpha^{\prime}$, in which case $T$ and $\widehat{\mathcal{O}}$ become $m^{2} \times m^{2}$ matrices. Upon iteration of (5) one finds,

$$
{ }_{N}\left\langle\alpha\left|\mathcal{O}_{n}\right| \alpha^{\prime}\right\rangle_{N}=\sum_{\beta}\left(T^{N-n} \widehat{\mathcal{O}}\right)_{\alpha \alpha^{\prime}, \beta \beta}
$$

More generally, the expectation value of a product of local operators is given by,

$$
{ }_{N}\left\langle\alpha\left|\mathcal{O}_{n_{1}}^{(1)} \mathcal{O}_{n_{2}}^{(2)} \cdots \mathcal{O}_{n_{r}}^{(r)}\right| \alpha^{\prime}\right\rangle_{N}
$$

$$
=\sum_{\beta}\left(T^{N-n_{1}} \widehat{\mathcal{O}}^{(1)} T^{n_{1}-n_{2}-1} \widehat{\mathcal{O}}^{(2)} \cdots T^{n_{r-1}-n_{r}-1} \widehat{\mathcal{O}}^{(r)}\right)
$$

where $N \geq n_{1}>n_{2}>\cdots>n_{r} \geq 1$. The matrix $T$ plays a very important role in the MPM. Eqs (5.96) imply that $T$ behaves as a shift operator by one lattice space. The basic properties of $T$ follow from the normalization condition (2) which can be expressed as,

$$
\sum_{\beta} T_{\alpha \alpha^{\prime}, \beta \beta}=\delta_{\alpha, \alpha^{\prime}}
$$

which implies that $T$ has a eigenvalue equal to 1 . Let us call $|v\rangle$ the right eigenvector corresponding to this eigenvalue. Eq.(10) can be written in matrix notation as,

$$
T|v\rangle=|v\rangle, \quad v_{\alpha \alpha^{\prime}}=\delta_{\alpha \alpha^{\prime}}
$$

On the other hand, let $\langle\rho|$ denote the left eigenvector of $T$ corresponding to the eigenvalue 1, i.e.

$$
\langle\rho| T=\langle\rho| \leftrightarrow \sum_{\alpha \alpha^{\prime}} \rho_{\alpha \alpha^{\prime}} T_{\alpha \alpha^{\prime}, \beta \beta^{\prime}}=\rho_{\beta \beta^{\prime}}
$$

A convenient normalization of $\langle\rho|$ is given by

$$
\langle\rho \mid v\rangle=1 \leftrightarrow \sum_{\alpha} \rho_{\alpha \alpha}=1
$$

For later use we shall diagonalize $T$ as follows,

$$
T=\sum_{p} x_{p}\left|v_{p}\right\rangle\left\langle\rho_{p}\right|, \quad\left\langle\rho_{p} \mid v_{p^{\prime}}\right\rangle=\delta_{p p^{\prime}}
$$

where $\left|v_{p}\right\rangle$ and $\left\langle\rho_{p}\right|$ are the right and left eigenvectors of $T$ with eigenvalue $x_{p} \quad\left(x_{1}=1,\left|v_{1}\right\rangle=|v\rangle,\left\langle\rho_{1}\right|=\langle\rho|\right)$. As a matter of fact all the remaining eigenvalues of $T$ are less than one, i.e. $\left|x_{p}\right|<1 \quad \forall p \neq 1$.

In the limit $N \rightarrow \infty$ one gets,

$$
\begin{gathered}
\lim _{N \rightarrow \infty}\left\langle\alpha\left|\mathcal{O}_{n_{1}}^{(1)} \mathcal{O}_{n_{2}}^{(2)} \cdots \mathcal{O}_{n_{r}}^{(r)}\right| \alpha^{\prime}\right\rangle_{N} \\
=\delta_{\alpha \alpha^{\prime}}\left\langle\rho\left|\widehat{\mathcal{O}}^{(1)} T^{n_{1}-n_{2}-1} \widehat{\mathcal{O}}^{(2)} \cdots T^{n_{r-1}-n_{r}-1} \widehat{\mathcal{O}}^{(r)}\right| v\right\rangle
\end{gathered}
$$

The delta function on the r.h.s. of this eq. means that the local operators $\widehat{\mathcal{O}}^{(n)}$ acting in the bulk, do not modify the boundary conditions associated to the various choices of $\alpha$.

Assuming that $T$ is invertible, one can rewrite eq.(15) in the following manner,

$$
\begin{aligned}
& \lim _{N \rightarrow \infty N}\left\langle\alpha\left|\mathcal{O}_{n_{1}}^{(1)} \mathcal{O}_{n_{2}}^{(2)} \cdots \mathcal{O}_{n_{r}}^{(r)}\right| \alpha^{\prime}\right\rangle_{N} \\
= & \delta_{\alpha \alpha^{\prime}}\left\langle\rho\left|\tilde{\mathcal{O}}^{(1)}\left(n_{1}\right) \tilde{\mathcal{O}}^{(2)}\left(n_{2}\right) \cdots \tilde{\mathcal{O}}^{(r)}\left(n_{r}\right)\right| v\right\rangle
\end{aligned}
$$

where $\tilde{\mathcal{O}}(n)$ is defined as

$$
\tilde{\mathcal{O}}(n)=T^{-n-1} \widehat{\mathcal{O}} T^{n}
$$

Observe that $\tilde{1}=1$. The r.h.s. of (16) is nothing but a spatial ordered product of local operators $\tilde{\mathcal{O}}(n)$, which is reminiscent of the radial ordered product that appears in $\beta \beta$ Conformal Field Theory. This connection supports the interpretation of $T$ as an euclidean version of the shift operator. Under this viewpoint the states $|v\rangle$ and $\langle\rho|$ appear as incoming $|0\rangle$ and outgoing vacua $\langle 0|$ that are left invariant by the shift operator $T$.

We have shown above that the MPM leads in the thermodynamic limit to a sort of discretized field theory characterized by a shift or spatial transfer operator $T$ and local operators $\tilde{\mathcal{O}}(n)$. We can now try to exploit these interpretation to extract some physical quantities.

First of all let us consider the correlator of two operators $\mathcal{O}^{(1)}\left(n_{1}\right)$ and $\mathcal{O}^{(2)}\left(n_{2}\right)$. From (14, 15) one has,

$$
\begin{gathered}
\left\langle\rho\left|\widehat{\mathcal{O}}^{(1)} T^{n_{1}-n_{2}-1} \widehat{\mathcal{O}}^{(2)}\right| v\right\rangle \\
=\sum_{p} x_{p}^{n_{1}-n_{2}-1}\left\langle\rho\left|\widehat{\mathcal{O}}^{(1)}\right| v_{p}\right\rangle\left\langle\rho_{p}\left|\widehat{\mathcal{O}}^{(2)}\right| v\right\rangle
\end{gathered}
$$

In the limit when $\left|n_{1}-n_{2}\right|>>1$ the sum over $p$ is dominated by the highest eigenvalue $\left|x_{p}\right|$ of $T$ for which the corresponding matrix elements $\left\langle\rho\left|\widehat{\mathcal{O}}^{(1)}\right| v_{p}\right\rangle$ and $\left\langle\rho_{p}\left|\widehat{\mathcal{O}}^{(2)}\right| v\right\rangle$ are non zero. If $x_{p}<1$ one gets a finite correlation lenght $\xi$ given by the formula,

$$
\xi=-1 / \ln \left|x_{p}\right|
$$

In the case where $\widehat{\mathcal{O}}^{(1)}$ and $\widehat{\mathcal{O}}^{(2)}$ are both the spin operator $\mathbf{S}$, it turns out that the matrix element $\langle\rho|\widehat{\mathbf{S}}| v\rangle$ vanishes, and hence the spin-spin correlator is short ranged 
with a finite spin correlation length $\xi$ given by the formula (19) with $\left|x_{p}\right|<1$. The finiteness of $\xi$ does indeed occur for MP ansatzs which preserve the rotational invariance. However if the latter is broken, as in a Neel like state, then $\xi$ may become infinite.

In section III we shall give a formula to compute $\xi$ in the case of rotational invariant MP ansatzs.

Another interesting application of (16) is provided by the computation of the string order parameter.

\section{String order parameter}

A spin 1 chain has a long range topological order (LRTO) characterized by a non vanishing value of a non local operator $g(\infty)$ defined as follows 10 ,

$$
\begin{gathered}
g(\infty)=\lim _{\ell \rightarrow \infty} g(\ell) \\
g(\ell)=\left\langle S^{z}(\ell) \prod_{k=1}^{\ell-1} e^{\pi \mathrm{i} S^{z}(k)} S^{z}(0)\right\rangle
\end{gathered}
$$

The AKLT state has $g_{A K L T}(\infty)=-(2 / 3)^{2}$, while the spin 1 antiferromagnetic spin chain has $g(\infty)=$ $-0.374325[1]$. From eq.(15) we deduce the following expression for 20 ,

$$
g(\ell)=\left\langle\rho\left|\widehat{S^{z}}\left(e^{\widehat{\mathrm{i} \pi S^{z}}}\right)^{\ell-1} \widehat{S^{z}}\right| v\right\rangle
$$

In appendix $\mathrm{C}$ we show that the operator $e^{\widehat{\mathrm{i} \pi S^{z}}}$ has an eigenvalue equal to 1 . Denoting by $\left|v^{\text {st }}\right\rangle$ and $\left\langle\rho^{\text {st }}\right|$ the associated right and left eigenvectors we obtain the following expression for the string order parameter

$$
g(\infty)=\left\langle\rho\left|\widehat{S^{z}}\right| v^{\mathrm{st}}\right\rangle\left\langle\rho^{\mathrm{st}}\left|\widehat{S^{z}}\right| v\right\rangle
$$

which suggests that $g(\infty)$ measures a sort of off-diagonal order.

For antiferromagnetic spin 1 ladders we shall see that the LRTO disappears and that the correlator (20) is short ranged with a finite correlation length $\xi^{\text {st }}$.

\section{Ground state energy density}

Let us suppose we have a translational invariant Hamiltonian of the form,

$$
H_{N}=\sum_{n=1}^{N} h_{n}^{(1)}+\sum_{n=1}^{N-1} h_{n, n+1}^{(2)}
$$

where $h^{(1)}$ is an on site (rung) operator while $h^{(2)}$ couples two nearest neighbour sites (rungs). We define the expectation value,

$$
E_{\alpha \alpha^{\prime}}^{N}={ }_{N}\left\langle\alpha\left|H_{N}\right| \alpha^{\prime}\right\rangle_{N}
$$

which can be computed recursively. From eqs.(115) one gets

$$
E_{\alpha \alpha^{\prime}}^{N}=\sum_{\beta \beta^{\prime}} T_{\alpha \alpha^{\prime}, \beta \beta^{\prime}} E_{\beta \beta^{\prime}}^{N-1}+\sum_{\beta} \widehat{h}_{\alpha \alpha^{\prime}, \beta \beta}, \quad(N \geq 2)
$$

where $\widehat{h}=\widehat{h}^{(1)}+\widehat{h}^{(2)}$. The hated representation of the site hamiltonian $h^{(1)}$ is given by eq $(7)$, while the hated representation of the hamiltonian $h^{(2)}$ is given by,

$$
\begin{gathered}
\widehat{h}_{\alpha \alpha^{\prime}, \beta \beta^{\prime}}^{(2)}=\sum_{\gamma \gamma^{\prime} s^{\prime} s} N-1, N\left\langle s_{2} s_{1}\left|h_{N-1, N}^{(2)}\right| s_{1}^{\prime} s_{2}^{\prime}\right\rangle_{N, N-1} \\
\times A_{\alpha, \gamma}^{*}\left[s_{1}\right] A_{\gamma, \beta}^{*}\left[s_{2}\right] A_{\alpha^{\prime}, \gamma^{\prime}}\left[s_{1}^{\prime}\right] A_{\gamma^{\prime}, \beta^{\prime}}\left[s_{2}^{\prime}\right]
\end{gathered}
$$

It should be clear from eqs. (77, 26) which is the hated representative of an operator involving an arbitrary number of sites. Eq.(25) can be conveniently written in matrix notation as

$$
\left|E^{N}\right\rangle=T\left|E^{N-1}\right\rangle+\widehat{h}|v\rangle, \quad(N \geq 2)
$$

where $\left|E^{N}\right\rangle$ is a vector with components $E_{\alpha \alpha^{\prime}}^{N}$. Iterating (27) one gets

$$
\left|E^{N}\right\rangle=\left(1+T+T^{2}+\cdots+T^{N-2}\right) \widehat{h}|v\rangle+T^{N-1}\left|E^{1}\right\rangle
$$

The geometric series in $T$ can be sumed up and due to the eigenvalue equal to 1 it contributes a term proportional to $N$, i.e.

$$
\lim _{N \rightarrow \infty} \frac{1}{N}\left|E^{N}\right\rangle=e_{\infty}|v\rangle
$$

This eq. implies that all the states $|\alpha\rangle_{N}$ have the same energy density in the thermodynamic limit, i.e. $E_{\alpha \alpha^{\prime}}^{N}=$ $\delta_{\alpha \alpha^{\prime}} e_{\infty}$. Hence $e_{\infty}$ can be identified with the GS energy per site for chains or per rung for ladders and it is given by,

$$
e_{\infty}=\langle\rho|\widehat{h}| v\rangle=\sum_{\alpha \alpha^{\prime} \beta} \rho_{\alpha \alpha^{\prime}} \widehat{h}_{\alpha \alpha^{\prime}, \beta \beta}
$$

This is the quantity one has to minimize respect to the MPM parameters.

The formalism presented above is closely related to the DMRG. Eventhough this relation is not the main subject of this paper we shall make some remarks ( see [7]).

\section{MPM versus the DMRG}

Let us suppose that we diagonalize $\rho$, as a $m \times m$ matrix, denoting its eigenvalues as $w_{\alpha}^{2}$, i.e.

$$
\rho_{\alpha \alpha^{\prime}}=w_{\alpha}^{2} \delta_{\alpha \alpha^{\prime}}
$$

The eigenvalue eq.(12) becomes then,

$$
\sum_{\alpha s} w_{\alpha}^{2} A_{\alpha \beta}[s] A_{\alpha \beta^{\prime}}^{*}[s]=\delta_{\beta \beta^{\prime}} w_{\beta}^{2}
$$


There is a close analogy between eqs(2) and (32), except for the fact that the order of the labels is exchanged. Given the tensor product decomposition $s \otimes \beta \rightarrow \alpha$, we shall assume that one can reverse the order between the states $\alpha$ and $\beta$ in terms of "charge conjugate states" $\alpha^{c}$ and $\beta^{c}$, as follows : $s \otimes \alpha^{c} \rightarrow \beta^{c}$. For example the charge conjugate of a state with spin $M$ is another state with spin $-M$. Using this concept we can impose the following symmetry condition [12],

$$
w_{\alpha} A_{\alpha \beta}[s]= \pm w_{\beta^{c}} A_{\beta^{c} \alpha^{c}}[s], \quad w_{\beta^{c}}=w_{\beta}
$$

which leads to the equivalence between eqs. (2) and (32).

The relation between the MPM and the DMRG is made clear by the construction of the GS of the superblock $B_{N} \bullet B_{N}^{R}$ in the following way,

$$
\begin{gathered}
\left|\psi_{0}\right\rangle=\sum \psi_{\alpha s \beta}\left|\alpha^{R}\right\rangle \otimes|s\rangle \otimes|\beta\rangle \\
\psi_{\alpha s \beta}=w_{\alpha} A_{\alpha \beta}[s]
\end{gathered}
$$

The density matrix that induces $\psi_{\alpha s \beta}$ on the block $B$, and which is obtained by tracing over the states in $\bullet B_{N}^{R}$, coincides with $\rho_{\alpha \alpha^{\prime}}=w_{\alpha}^{2} \delta_{\alpha \alpha^{\prime}}$.

Condition (33) guarantees that $\left|\psi_{0}\right\rangle$ is a state invariant under the parity transformation that interchanges the blocks $B$ and $B_{N}^{R}$, while leaving invariant the site $\bullet$.

It is interesting to observe that the MPM leads to a superblock of the form $B_{N} \bullet B_{N}^{R}$, rather than to the standard superblock $B_{N} \bullet \bullet B_{N}^{R}$ []].

\section{III) THE MPM APPLIED TO SPIN LADDERS}

In this section we shall apply the MPM to the 2-leg spin ladder with a spin $S$ at each site of the chain. The collection $|\alpha\rangle_{N}$ will be given by the set $|J M\rangle_{N}\left(J \leq J_{\max }\right)$ of states with total spin $J$ and third component $M$. For the sake of simplicity we have only considered one state per angular momenta $J$ and $M$. This will allow us to show more clearly the analytic structure of the MPM, which can later on be numerically improved by considering multiplicity. This has already been done in the case of spin chains in references [5,7].

The states added at each step of the MPM are the ones that appear in the tensor product decomposition of two spin $S$ irreps, i.e. $S \otimes S=0 \oplus 1 \oplus \cdots \oplus 2 S$. These states are labelled by $|\lambda \mu\rangle$ where $\lambda=0, \cdots, 2 S$ is the total spin and $\mu=-\lambda, \ldots, \lambda$ is its third component.

Using these notations we propose the following recurrence relation for the states $|J M\rangle_{N}$,

$$
\left|J_{1} M_{1}\right\rangle_{N}=\sum_{\lambda J_{2}} A_{J_{1} J_{2}}^{\lambda}\left|\left(\lambda J_{2}\right), J_{1} M_{1}\right\rangle_{N}
$$

where

$$
\left|\left(\lambda J_{2}\right), J_{1} M_{1}\right\rangle_{N}
$$

$$
=\sum_{\mu}\left\langle\lambda \mu, J_{2} M_{2} \mid \lambda J_{2}, J_{1} M_{1}\right\rangle|\lambda \mu\rangle_{N} \otimes\left|J_{2} M_{2}\right\rangle_{N-1}
$$

In (36) the quantity $\left\langle\lambda \mu, J_{2} M_{2} \mid \lambda J_{2}, J_{1} M_{1}\right\rangle$ is the Clebsch-Gordan coefficient corresponding to the decomposition $\lambda \otimes J_{2} \rightarrow J_{1}$. Comparing eqs.(11) and (35) we obtain the following relation between the symbols $A_{J_{1} M_{1}, J_{2} M_{2}}[\lambda \mu]$ and the rotational invariant symbols $A_{J_{1} J_{2}}^{\lambda}$

$$
A_{J_{1} M_{1}, J_{2} M_{2}}[\lambda \mu]=A_{J_{1} J_{2}}^{\lambda}\left\langle\lambda \mu, J_{2} M_{2} \mid \lambda J_{2}, J_{1} M_{1}\right\rangle
$$

The use of rotational invariant basis reduces considerably the number of independent variational parameters and consequently increases the power of the MPM [5, [7].

The variational parameters $A_{J_{1} J_{2}}^{\lambda}$ are subject to the CG condition,

$$
A_{J_{1} J_{2}}^{\lambda}=0 \text { unless }\left|\lambda-J_{2}\right| \leq J_{1} \leq\left|\lambda+J_{2}\right|
$$

Using (37) and the orthogonality properties of the CG coefficients, the normalization conditions (2) become,

$$
\sum_{\lambda, J_{2}}\left|A_{J_{1} J_{2}}^{\lambda}\right|^{2}=1, \quad \forall J_{1}
$$

At this point we can just take eq.(37) and plug it into the corresponding formulas of section II in order to derive expectation values, the GS energy density, etc in terms of $A_{J_{1} J_{2}}^{\lambda}$. There is however a more efficient way to do this by using group theory. The application of the WignerEckart theorem will allow us to express all the results in terms of reduced matrix elements of the operators involved as well as the $6 j$-symbols. In our derivations we shall follow the same steps as in section II, leaving the technical details to appendix B.

\section{Correlators of Invariant Tensors}

Let us denote by $\mathcal{O}^{(k)}$ an irreducible tensor of total angular momentum $k$, whose components are labeled by $\mathcal{O}_{M}^{(k)}, M=-k, \ldots, k$. The spin operators $\mathbf{S}$ correspond to $k=1$. Let us suppose we have two irreducible tensors with the same total angular momenta $k, \mathcal{O}^{(k, A)}(n)$ and $\mathcal{O}^{(k, B)}(m)$, acting at the positions $n$ and $m(N \geq n>$ $m \geq 1$ ) of the ladder. The scalar product of these two operators is defined as

$$
\begin{gathered}
\mathcal{O}^{(k, A)}(n) \cdot \mathcal{O}^{(k, B)}(m) \\
=\sum_{M=-k}^{k}(-1)^{-M} \mathcal{O}_{M}^{(k, A)}(n) \cdot \mathcal{O}_{-M}^{(k, B)}(m)
\end{gathered}
$$

The basic result we derive in appendix $B$ is,

$$
{ }_{N}\left\langle J_{1} M\left|\mathcal{O}^{(k, A)}(n) \cdot \mathcal{O}^{(k, B)}(m)\right| J_{1} M\right\rangle_{N}
$$




$$
=\sum_{J_{2}, \ldots, J_{7}} T_{J_{1} J_{2}}^{N-n} \widehat{\mathcal{O}}_{J_{2}, J_{3} J_{4}}^{(k, A)}\left(T_{k}^{n-m-1}\right)_{J_{3} J_{4}, J_{5} J_{6}} \widehat{\mathcal{O}}_{J_{5} J_{6}, J_{7}}^{(k, B)}
$$

where $T$ and $T^{(k)}$ are defined as,

$$
\begin{gathered}
T_{J_{1}, J_{2}}=\sum_{\lambda}\left(A_{J_{1} J_{2}}^{\lambda}\right)^{*} A_{J_{1} J_{2}}^{\lambda} \\
\left(T_{k}\right)_{J_{1} J_{2}, J_{3} J_{4}}=\sum_{\lambda}\left(A_{J_{1} J_{3}}^{\lambda}\right)^{*} A_{J_{2} J_{4}}^{\lambda} \\
\times(-1)^{\lambda+k+J_{1}+J_{4}} \sqrt{\left(2 J_{1}+1\right)\left(2 J_{2}+1\right)}\left\{\begin{array}{lll}
J_{3} & J_{1} & \lambda \\
J_{2} & J_{4} & k
\end{array}\right\}
\end{gathered}
$$

while $\widehat{\mathcal{O}}^{(k, B)}$ and $\widehat{\mathcal{O}}^{(k, B)}$ are defined in Appendix B.

Eq.(41) is the invariant version of (9) involving only two operators. In order to obtain the thermodynamic properties of (41) we use the properties of the transfer operator $T$. The normalization conditions (39) imply the following conditions on $T$,

$$
\sum_{J_{2}} T_{J_{1}, J_{2}}=1, \quad \forall J_{1}
$$

Let us call $\rho_{J}$ the left eigenvector of $T_{J_{1}, J_{2}}$ with eigenvalue 1 , i.e.

$$
\sum_{J_{1}} \rho_{J_{1}} T_{J_{1}, J_{2}}=\rho_{J_{2}}
$$

Using eqs.(44, 45) into (41) and taking $N>>1$ we get

$$
\begin{aligned}
\lim _{N \rightarrow \infty} & { }_{N}\left\langle J_{1} M\left|\mathcal{O}^{(k, A)}(n) \cdot \mathcal{O}^{(k, B)}(m)\right| J_{1} M\right\rangle_{N} \\
= & \left\langle\rho\left|\widehat{\mathcal{O}}^{(k, A)} T_{k}^{n-m-1} \widehat{\mathcal{O}}^{(k, B)}\right| v\right\rangle
\end{aligned}
$$

where we use a matrix notation in $J$-space with the convention $v_{J}=1, \forall J$. From eq.(46) we deduce that the correlation length associated to the scalar product of two irreducible operators with angular momentum $k$, is given by the highest eigenvalue of the matrix $T_{k}$ defined in (43). The spin-spin correlation length is obtained by looking at the highest absolute eigenvalue of $T_{1}$.

\section{Ground state energy density}

The Hamiltonian of the 2-leg ladder has the form proposed in (23) where $h^{(1)}$ is the rung Hamiltonian and $h^{(2)}$ is the leg Hamiltonian,

$$
\begin{gathered}
h_{n}^{(1)}=J_{\perp} \mathbf{S}_{1}(n) \cdot \mathbf{S}_{2}(n) \\
h_{n, n+1}^{(2)}=J_{\|}\left(\mathbf{S}_{1}(n) \cdot \mathbf{S}_{1}(n+1)+\mathbf{S}_{2}(n) \cdot \mathbf{S}_{2}(n+1)\right)
\end{gathered}
$$

$\mathbf{S}_{a}(n)$ is a spin $S$ operator acting on the $n=1, \cdots, N$ rung and the $a=1,2$ leg of the ladder.

As in (24) we define the expectation value of the ladder Hamiltonian,

$$
E_{J}^{N}={ }_{N}\left\langle J M\left|H_{N}\right| J M\right\rangle_{N}
$$

Using (35) we find

$$
E_{J_{1}}^{N}=\sum_{J_{2}}\left(T_{J_{1}, J_{2}} E_{J_{2}}^{N-1}+\widehat{h}_{J_{1}, J_{2}}\right), \quad(N \geq 2)
$$

where $\widehat{h}=\widehat{h}^{(1)}+\widehat{h}^{(2)}\left(\widehat{h}^{(1)}\right.$ and $\widehat{h}^{(2)}$ can be found in appendix B).

Iterating eq.(50) and using the properties of the matrix (43) $T$ we can inmediately get the large $N$ limit of the energy (49),

$$
\lim _{N \rightarrow \infty} \frac{1}{N} E_{J}^{N}=e_{\infty}, \quad \forall J
$$

where the GS energy density is given by,

$$
e_{\infty}=\langle\rho|\widehat{h}| v\rangle=\sum_{J_{1}, J_{2}} \rho_{J_{1}} \widehat{h}_{J_{1} J_{2}}
$$

At this point let us summarize the main steps of the MP algorithm hereby proposed,

- Solve the normalization conditions (39) expressing $A_{J_{1} J_{2}}^{\lambda}$ in terms of a set of linearly independent variational parameters.

- Find the eigenvector $\rho_{J}$ of the matrix $T$.

- Minimize the GS energy density (52) with respect to the independent variational parameters.

We will now comment on how these three steps can be implemented.

\section{Solution of the normalization conditions}

We shall suppose in the rest of the paper that the parameters $A_{J_{1} J_{2}}^{\lambda}$ are all real. Hence the normalization conditions

$$
\sum_{\lambda, J_{2}}\left(A_{J_{1} J_{2}}^{\lambda}\right)^{2}=1, \quad \forall J_{1}
$$

imply that the set $\left\{A_{J_{1} J_{2}}^{\lambda}\right\}$ for $J_{1}$ fixed are the coordinates of a sphere whose dimension depends on the allowed values of $J$ and the CG conditions (38). Let us call $A_{J_{1}}^{\max }$ the highest coordinate, in absolute value, i.e.

$$
A_{J_{1}}^{\max }=A_{J_{1} L_{0}}^{\lambda_{0}} \text { such that }\left|A_{J_{1} L_{0}}^{\lambda_{0}}\right| \geq\left|A_{J_{1} J_{2}}^{\lambda}\right| \forall \lambda, J_{2}
$$

If $A_{J_{1}}^{\max }>0\left(\right.$ resp. $\left.A_{J_{1}}^{\max }<0\right)$ we can think of it as the north ( resp. south) pole of a sphere, whose neaghbourhood can be described by the stereographic coordinates,

$$
x_{J_{1} J_{2}}^{\lambda}=A_{J_{1} J_{2}}^{\lambda} / A_{J_{1}}^{\max }, \quad\left|x_{J_{1} J_{2}}^{\lambda}\right| \leq 1
$$

Notice that $x_{J_{1} L_{0}}^{\lambda_{0}}=1$. The remaining coordinates are the independent variational parameters used in the minimization of the GS energy. The solution of the constraint (53) finally reads, 


$$
A_{J_{1} J_{2}}^{\lambda}=\epsilon_{J_{1}} x_{J_{1} J_{2}}^{\lambda}\left(\sum_{\lambda^{\prime}, J_{2}^{\prime}}\left(x_{J_{1} J_{2}^{\prime}}^{\lambda^{\prime}}\right)^{2}\right)^{-1 / 2}, \epsilon_{J}= \pm 1
$$

where $\epsilon_{J_{1}}=1(-1)$ corresponding to the north ( south ) pole of the above mentioned sphere.

\section{Determination of $\rho_{J}$}

The solution of the eigenvalue problem of eq.45) can be done numerically. However for a ladder with spin $S=1 / 2$ it can also be solved analytically which will allow us to make some considerations on the nature of $\rho_{J}$. In the case where $S=1 / 2$ the allowed values for $\lambda$ are 0 and 1 . Hence the unique non-vanishing entries of $A_{J_{1} L_{2}}^{\lambda}$ are $A_{J J}^{0}, A_{J J}^{1}, A_{J J+1}^{1}$ and $A_{J J-1}^{1}$. Similarly from eq. (42) the non-vanishing entries of $T$ are $T_{J, J}, T_{J, J+1}$ and $T_{J, J-1}$. The set of eqs. we have therefore to solve read explicitely as,

$$
\begin{gathered}
T_{J, J}+T_{J, J+1}+T_{J, J-1}=1 \\
\rho_{J} T_{J, J}+\rho_{J+1} T_{J+1, J}+\rho_{J-1} T_{J-1, J}=\rho_{J} \\
\sum_{J=0}^{J_{\max }} \rho_{j}=1
\end{gathered}
$$

The solution of these eqs. is given by,

$$
\rho_{J}=\frac{u_{J}}{\sum_{L} u_{L}}
$$

where

$$
u_{0}=1, \quad u_{J}=\prod_{L=0}^{J} \frac{T_{L, L+1}}{T_{L+1, L}} \quad(J>0)
$$

we are assuming that $J=0, \cdots, J_{\max }$.

Eqs. $(58,59)$ imply that $\rho_{J}$ is always positive, in agreement with the Perron-Frobenius theorem applied to the matrix $T$, whose entries are all non-negative. In ref. [7] it was shown that the values of $\rho_{J}$ are intimately related to the eigenvalues of the density matrix that appear in the DMRG. These and other facts suggest that the MPM is in fact equivalent to the DMRG, specially when the number of states kept $m$ becomes large.

This completes the presentation of the formalism.

\section{IV) NUMERICAL RESULTS}

In this section we shall apply the MPM to five different spin ladders, corresponding to different choices of the spin $S$ and signs of the coupling constants $J_{\|}$and $J_{\perp}$. We shall denote every of these ladders as $A A_{S}, A F_{S}$, and $F A_{S}$ where $A$ and $F$ stands for antiferromagnetic or ferromagnetic couplings. Thus for example $A F_{S}$ is a spin
S ladder with antiferromagnetic couplings along the legs and ferromagnetic couplings along the rungs. With these notations we will study below the following cases: $A A_{1 / 2}$, $A F_{1 / 2}, F A_{1 / 2}, A A_{1}$ and $A A_{3 / 2}$. Within each case we will highlight a particular aspect, which the MPM helps to clarify.

\section{$A A_{1 / 2}$-ladder: The dimer-RVB state}

This is the most studied spin ladder. Its properties are well known and can be summarized as follows. In the weak coupling regime, i.e. $J_{\perp}<<J_{\|}$, the gapless spin $1 / 2$ chains become massive by the interchain coupling which is a relevant operator of dimension 1 13 15. The magnitude of the gap is proportional to $J_{\perp}$. In the intermediate coupling regime, i.e. $J_{\perp} \simeq J_{\|}$the spin ladder can be mapped into the $O(3)$ non linear sigma model (NLSM) with no topological term 16 18]. This model is known to have a spin gap. From numerical studies the magnitude of the spin gap $\Delta$ and the spin correlation length, in the isotropic case $J_{\perp}=J_{\|}=J$, are given by $\Delta=0.502 \mathrm{~J}$ and $\xi=3.2$ respectively 19 22]. In the strong coupling regime $J_{\perp}>>J_{\|}$, the most appropiate physical picture of the GS and excitations is given by the RVB scenario proposed in [20], and supported by DMRG [20], mean field [23] and variational calculations [24]. In the latter work a recurrent variational ansatz (RVA) was proposed to generate the dimer-RVB and generalizations of it. The RVA method is a MPM based on $2^{\text {nd }}$ and higher order recurrent relations, while the standard MPM is based on a $1^{\text {st }}$ order relation. We shall see below that the MPM applied to ladders essentially contains the RVA, and that the numerical results are improved.

Let us first consider the case where the MPM states $|J M\rangle_{N}$ are choosen to be a singlet and a triplet, i.e.. $J=0$ and 1. In this case eq. (35) is depicted in fig. 1. There are a priori 5 non vanishing MP parameters subjected to 2 normalization constraints, leaving a total of 3 independent parameters.

Fig.2 shows $A_{J_{1} J_{2}}^{\lambda}$ as functions of $x=J_{\|} / J_{\perp}$. In the whole range of coupling constants the most important amplitudes are $A_{00}^{0}$ and $A_{10}^{1}$ while $A_{11}^{1}$ is essentially zero. The latter amplitude correspond to having only a single bond among two rungs ( see fig. 1), which is forbidden in the dimer-RVB picture of refs. 20] and [24].

In table 1 we show the GS energy density obtained with the MPM for $J_{\max }=1$ and 2 , together with the RVA, mean field and Lanczos results. In table 2 we give the spin correlation length computed with the MPM and the RVA.

There is an appreciable improvement in the numerical results of the MPM respect to the RVA, specially for the spin correlation length. 


\begin{tabular}{cccccc}
\hline \hline$J_{\|} / J_{\perp}$ & $J_{\max }=1$ & $J_{\max }=2$ & RVA & \multicolumn{2}{c}{ Mean Field Lanczos } \\
\hline \hline 0.0 & 0.375000 & 0.375000 & 0.375000 & 0.375000 & \\
\hline 0.2 & 0.383199 & 0.383199 & 0.383195 & 0.382548 & \\
\hline 0.4 & 0.409607 & 0.409608 & 0.409442 & 0.405430 & \\
\hline 0.6 & 0.453509 & 0.453513 & 0.45252 & 0.442424 & \\
\hline 0.8 & 0.510504 & 0.510523 & 0.507909 & 0.489552 & \\
\hline 1.0 & 0.575924 & 0.575970 & 0.571314 & 0.542848 & 0.578 \\
\hline 1.25 & 0.664776 & 0.664867 & 0.657551 & 0.614473 & 0.6687 \\
\hline 1.66 & 0.819656 & 0.819834 & 0.808438 & 0.738360 & 0.8333 \\
\hline 2.5 & 1.152056 & 1.152416 & 1.13384 & 1.002856 & 1.18 \\
\hline 5.0 & 2.172002 & 2.172878 & 2.13608 & & 2.265 \\
\hline \hline
\end{tabular}

Table 1. GS energy per site $-e_{\infty} / 2 J_{\perp}$ of the ladder $A A_{1 / 2}$. The first two columns are the MPM results.

The RVA results are obtained with a third order recursion formula 24]. The mean field and Lanczos results have been obtained in references [23] and [25] respectively.

\begin{tabular}{cccc}
\hline \hline$J_{\|} / J_{\perp}$ & $J_{\max }=1$ & $J_{\max }=2$ & RVA \\
\hline \hline 0.0 & 0.00000 & 0.0000 & 0.000000 \\
\hline 0.2 & 0.5300 & 0.5303 & 0.437166 \\
\hline 0.4 & 0.8057 & 0.8081 & 0.608323 \\
\hline 0.6 & 1.0652 & 1.0740 & 0.751286 \\
\hline 0.8 & 1.2753 & 1.2945 & 0.866958 \\
\hline 1.0 & 1.4282 & 1.4593 & 0.959249 \\
\hline 1.25 & 1.5572 & 1.6018 & 1.04877 \\
\hline 1.66 & 1.6802 & 1.7413 & 1.15205 \\
\hline 2.5 & 1.7903 & 1.8698 & 1.26951 \\
\hline 5.0 & 1.8747 & 1.9711 & 1.38532 \\
\hline \hline
\end{tabular}

Table 2 Spin correlation length of the ladder $A A_{1 / 2}$. The first two columns are the MPM results. The RVA results are those of ref 24.

\section{$A F_{1 / 2}$-ladder: Relation with the spin 1 chain}

The ladder with magnetic structure $A F$ is interesting because it is intimately related to the spin 1 chain [26]. This relation can be clearly seen in the strong coupling limit $-J_{\perp}>>J_{\|}$, since it leads to an effective spin 1 on every rung, which are effectively coupled antiferromagnetically along the legs. The effective Hamiltonian can be derived from (47,48) and reads [26],

$$
H_{\mathrm{eff}}^{\text {ladder }}=-\frac{1}{4}\left|J_{\perp}\right| N+\frac{1}{2} J_{\|} \sum_{n} \mathbf{S}_{\mathrm{eff}} \cdot \mathbf{S}_{\mathrm{eff}}
$$

where $\mathbf{S}_{\text {eff }}(n)=\mathbf{S}_{1}(n)+\mathbf{S}_{2}(n)$ is the spin 1 operator acting on the $n^{\text {th }}$ rung. The term proportional to $J_{\perp}$ comes from the rung Hamiltonian when diagonalized in the spin 1 sector. This eq. implies the following relation between the energies per site of the $A F$-ladder and the spin 1 chain,

$$
e_{\infty}^{A F}=-\frac{1}{8}\left|J_{\perp}\right|+\frac{1}{4} J_{\|} e_{\infty}^{\mathrm{eff}}
$$

In table 3 we give the GS energies of the ladder parametrized in terms of the effective energy $e_{\infty}^{\text {eff }}$. We also give the spin correlation length. We have made two choices of MP states $|J M\rangle_{N}$. One for which $J=0$ and 1 and the other for which $J=1 / 2$ and $3 / 2$. The latter one corresponds to having a single spin $1 / 2$ at the boundary of the ladder. These two choices have an analogue for the spin 1 chain. For integer $J^{\prime} s$ the MP parameters do not vary in the whole interval $0<J_{\|}<1.66$ and take the values,

$$
\begin{gathered}
A_{00}^{0}=A_{11}^{0}=0.000, A_{01}^{1}=1.000 \\
A_{10}^{1}=-0.577, A_{11}^{1}=0.816
\end{gathered}
$$

For half-integer $J^{\prime} s$ the MP parameters do not vary in the whole interval $0<J_{\|}<5$ and take the values,

$$
\begin{gathered}
A_{\frac{1}{2} \frac{1}{2}}^{0}=A_{\frac{3}{2} \frac{3}{2}}^{0}=0.000, A_{\frac{1}{2} \frac{1}{2}}^{1}=0.989 \\
A_{\frac{1}{2} \frac{3}{2}}^{1}=0.148, A_{\frac{3}{2} \frac{1}{2}}^{1}=-0.953, A_{\frac{3}{2} \frac{3}{2}}^{1}=-0.303
\end{gathered}
$$

Note that for half integers $J$ there is one more variational parameter. The most important amplitudes indeed correspond to the formation of an AKLT state with a single bond connecting every effective spin 1 .

\begin{tabular}{ccccc}
\hline$-J_{\|} / J_{\perp}$ & $-e_{\infty}^{\text {eff,int }}$ & $-e_{\infty}^{\text {eff,half }}$ & $\xi^{\text {int }}$ & $\xi^{\text {half }}$ \\
\hline \hline$(0.0,1.66)$ & 1.333333 & 1.399659 & 0.910 & 2.5997 \\
\hline 2.5 & 1.363970 & 1.399659 & 1.9682 & 2.5997 \\
\hline 5.0 & 1.498539 & 1.399659 & 1.9607 & 2.5997 \\
\hline
\end{tabular}

Table 3. The exact values of the GS energy density of a spin 1 chain and its correlation length are given by

$$
e_{\infty}=-1.4014845 \text { and } \xi=6.03 \text { 11. }
$$

The values of $e_{\infty}^{\text {eff }}$ and $\xi$ in the integer $J$ case coincide with those of the AKLT state, while the ones of the half-integer $J$ case coincide with those obtained with a MPM method applied to the spin 1 chain [7] where one keeps two MPM states with $J=1 / 2$ and $3 / 2$. These results provide additional support for the equivalence between the $A F_{1 / 2}$ ladder and the spin 1 chain in the strong and intermediate coupling regimes observed previously by other authors [26].

$$
F A_{1 / 2} \text {-ladder }
$$

In the strong coupling regime the ladders $F A_{1 / 2}$ and $A F_{1 / 2}$ have similar GS energies and correlation lengths ( see tables 1, 2 and 4). The MP parameters display also a similar behaviour although some of them are interchanged ( see figures 2 and 3 ). The physical reason of this is the common GS in the case where $J_{\|}=0$, given by the coherent superposition of valence bonds in the rungs. 


\begin{tabular}{ccc}
\hline$-J_{\|} / J_{\perp}$ & $-e_{\infty} / 2 J_{\perp}$ & $\xi$ \\
\hline \hline 0.0 & 0.375000 & 0.0000 \\
\hline 0.2 & 0.381754 & 0.5140 \\
\hline 0.4 & 0.399295 & 0.7577 \\
\hline 0.6 & 0.424396 & 1.010 \\
\hline 0.8 & 0.454891 & 1.277 \\
\hline 1.0 & 0.489324 & 1.554 \\
\hline 1.25 & 0.536374 & 1.895 \\
\hline 1.66 & 0.619895 & 2.381 \\
\hline 2.5 & 0.803434 & 2.992 \\
\hline 5.0 & 1.376973 & 3.520 \\
\hline
\end{tabular}

Table 4. GS energy per site and correlation length of the ladder $F A_{1 / 2}$.

The relation between $F A_{1 / 2}$ and $A F_{1 / 2}$ is part of a more general relation involving also the ladder $A A_{1 / 2}$ and can be established by means of a type of transformations called dualities in ref. [29].

\section{Duality properties of spin ladders}

On a 2-leg ladder one can define 3 types of dualities called $U, T$ and $S$, which mix or leave invariant the ladder's magnetic structures $A A, A F$ and $F A$ [29].

The $U$ duality maps a Hamiltonian with couplings constants $J_{\|}, J_{\perp}$ into a ladder with couplings constants $J_{\|}^{U}, J_{\perp}^{U}$ where,

$$
\begin{gathered}
J_{\|}^{U}=J_{\|}\left\langle\mathbf{S}_{1}(n) \cdot \mathbf{S}_{1}(n+1)\right\rangle /\left\langle\mathbf{S}_{1}(n) \cdot \mathbf{S}_{2}(n+1)\right\rangle \\
J_{\perp}^{U}=J_{\perp}
\end{gathered}
$$

Under $U$ the leg-bonds are transformed into diagonal ones while the rung-bonds are left invariant. The signs of $\left\langle\mathbf{S}_{1}(n) \cdot \mathbf{S}_{1}(n+1)\right\rangle$ and $\left\langle\mathbf{S}_{1}(n) \cdot \mathbf{S}_{2}(n+1)\right\rangle$ are determined by those of $J_{\|}$and $J_{\perp}$ respectively. Thus $U$ acts on the magnetic structures as follows,

$$
\begin{array}{ccc}
A A & \stackrel{U}{\rightarrow} & F A \\
J_{\|}(A A)>0 & \rightarrow & J_{\|}^{U}(F A)<0 \\
J_{\perp}(A A)>0 & \rightarrow & J_{\perp}^{U}(F A)>0 \\
A F & \stackrel{U}{\rightarrow} & A F \\
J_{\|}(A F)>0 & \rightarrow & J_{\|}^{U}(A F)>0 \\
J_{\perp}(A F)<0 & \rightarrow & J_{\perp}^{U}(A F)<0
\end{array}
$$

In fig.4 we show $J_{\|}^{U}(F A)$ and $J_{\|}^{U}(A F)$ as functions of $J_{\|}(A A)$ and $J_{\|}(A F)$ respectively.

The GS energy density of the ladder with coupling constants $J_{\|}^{U}, J_{\perp}^{U}$ is a lower bound of the original GS energy [29], i.e.

$$
\begin{aligned}
& e_{\infty}\left(J_{\|}^{U}(F A), J_{\perp}^{U}(F A)\right) \leq e_{\infty}\left(J_{\|}(A A), J_{\perp}(A A)\right) \\
& e_{\infty}\left(J_{\|}^{U}(A F), J_{\perp}^{U}(A F)\right) \leq e_{\infty}\left(J_{\|}(A F), J_{\perp}(A F)\right)
\end{aligned}
$$

In fig. 5 we show the validity of these inequalities, which in the strong coupling limit almost become identities. In fig. 6 we show the correlation legths for both $A A$ and the transformed $F A$ ladders. Again in the strong coupling limit they become very close.

The $T$ transformation consists in the replacement of the vertical bonds by diagonal ones, i.e.

$$
\begin{gathered}
J_{\|}^{T}=J_{\|} \\
J_{\perp}^{T}=J_{\perp}\left\langle\mathbf{S}_{1}(n) \cdot \mathbf{S}_{1}(n+1)\right\rangle /\left\langle\mathbf{S}_{1}(n) \cdot \mathbf{S}_{2}(n+1)\right\rangle
\end{gathered}
$$

which leads to the following action on magnetic structures,

$$
\begin{array}{clc}
A A & \stackrel{T}{\rightarrow} & A F \\
J_{\|}(A A)>0 & \rightarrow & J_{\|}^{T}(A F)>0 \\
J_{\perp}(A A)>0 & \rightarrow & J_{\perp}^{T}(A F)<0 \\
& & \\
F A & \stackrel{T}{\rightarrow} & F A \\
J_{\|}(F A)<0 & \rightarrow & J_{\|}^{T}(F A)<0 \\
J_{\perp}(F A)>0 & \rightarrow & J_{\perp}^{T}(F A)>0
\end{array}
$$

In fig. 7 we plot the energies associated to the $F A$ ladder and its $T$ transformed, which satisfies the inequality,

$$
e_{\infty}\left(J_{\|}^{T}(F A), J_{\perp}^{T}(F A)\right) \leq e_{\infty}\left(J_{\|}(F A), J_{\perp}(F A)\right)
$$

The convergence of both curves in the weak coupling is in agreement with the bosonization arguments employed in [29].

Finally the $S$ transformation is defined by the replacement of vertical bonds by horizontal ones and viceversa,

$$
\begin{aligned}
& J_{\|}^{S}=\frac{1}{2} J_{\perp}\left\langle\mathbf{S}_{1}(n) \cdot \mathbf{S}_{2}(n)\right\rangle /\left\langle\mathbf{S}_{1}(n) \cdot \mathbf{S}_{1}(n+1)\right\rangle \\
& J_{\perp}^{S}=2 J_{\|}\left\langle\mathbf{S}_{1}(n) \cdot \mathbf{S}_{1}(n+1)\right\rangle /\left\langle\mathbf{S}_{1}(n) \cdot \mathbf{S}_{2}(n)\right\rangle
\end{aligned}
$$

The factors 2 and $1 / 2$ are explained by the fact that there are two leg-bonds for each rung-bond. Eqs.(72) imply,

$$
\begin{array}{ccc}
A F & \stackrel{S}{\rightarrow} & F A \\
J_{\|}(A F)>0 & \rightarrow & J_{\|}^{S}(F A)<0 \\
J_{\perp}(A F)<0 & \rightarrow & J_{\perp}^{S}(F A)>0 \\
& & \\
A A & \stackrel{S}{\rightarrow} & A A \\
J_{\|}(A A)>0 & \rightarrow & J_{\|}^{S}(A A)>0 \\
J_{\perp}(A A)>0 & \rightarrow & J_{\perp}^{S}(A A)>0
\end{array}
$$

In fig. 8 we plot the energies of the $A A$ ladder and its transformed which satisfy the inequality,

$$
e_{\infty}\left(J_{\|}^{S}(A A), J_{\perp}^{S}(A A)\right) \leq e_{\infty}\left(J_{\|}(A A), J_{\perp}(A A)\right)
$$

Note that in the region $J_{\|} \sim J_{\perp}$ both energies get very closed. Fig. 9 shows the spin correlation length for the AA ladder and its $S$ transformed, displaying the same pattern as fig. 8. In summary we have found further numerical evidence of the duality properties of the 2-leg ladder proposed in 29]. 


\section{$A A_{1}$-ladder: Short-range string order}

In table 5 we show the GS energy density and the spincorrelation length of the ladder $A A_{1}$. Observe that the correlation length is longer that the one of the spin $1 / 2$ ladder.

\begin{tabular}{ccc}
\hline$J_{\|} / J_{\perp}$ & $-e_{\infty} / 2 J_{\perp}$ & $\xi$ \\
\hline \hline 0.0 & 1.000000 & .00000 \\
\hline 0.2 & 1.055719 & 1.0114 \\
\hline 0.4 & 1.206557 & 1.8318 \\
\hline 0.6 & 1.407358 & 2.3852 \\
\hline 0.8 & 1.631166 & 2.6762 \\
\hline 1.0 & 1.867327 & 2.8227 \\
\hline 1.25 & 2.172905 & 2.9042 \\
\hline 1.66 & 2.688880 & 2.9286 \\
\hline
\end{tabular}

Table 5. GS energy per site and correlation length of the $A A_{1}$ ladder.

As mentioned in the introduction a spin 1 chain has a long range topological order (LRTO) characterized by a non-vanishing $g(\infty)$. In appendix $\mathrm{C}$ we give an analytical expression for $g(\infty)$ in terms of the MP parameters of the spin 1 chain.

However when two spin 1 chains are coupled antiferromagnetically the LRTO disappears and the string order parameter $g(\ell)$ decays exponentially as $e^{-\ell / \xi^{\text {st }}}$. We call $\xi^{\text {st }}$ the string correlation length, and its value together with the spin correlation length are shown in fig. 10 as functions of the ratio $J_{\|} / J_{\perp}$. In the weak coupling limit where $J_{\|} / J_{\perp} \rightarrow \infty$ we expect $\xi^{\text {st }}$ to diverge, recovering in that way the LRTO of the uncoupled chains. The value of $\xi^{\text {st }}$ is obtained by the formula (19) with $x_{p}$ the highest eigenvalue of the operator $e^{\widehat{i \pi S_{1}^{z}}}$ (see appendix $\mathrm{C}$ ).

An intuitive way to understand the breaking of the LRTO is given by the AKLT picture of ref [3]. An AKLT state is a valence bond state where every spin 1 is represented as a symmetrized product of two spins $1 / 2$, and such that every of these "elementary" spins is linked by a bond to one of the spins $1 / 2$ on its neighbours. In this way all the spins of the chain are connected by a sucession of nearest neighbour links. When we couple antiferromagnetically two spin 1 chains there is the possibility that two parallel bonds along the legs become two parallel bonds along the rungs as shown in fig. 11. Thus the two infinite parallel arrays of connected bonds, characteristic of the uncoupled chains, effectively breaks into a collection of fluctuating islands whose size is of the order of $\xi^{\text {st }}$. Everyone of these islands is a sort of closed spin 1 chain ( fig.11).

The finite value of $\xi^{\text {st }}$ at the origin of fig.10 is due to the fact that $e^{i \pi S_{1}^{z}}$ has indeed a finite value when computed on the singlet formed by two spins 1 on a rung,

$$
\left\langle e^{i \pi S_{1}^{z}}\right\rangle_{\text {rung }}=\sum_{m= \pm 1,0}(-1)^{m}\langle 1 m 1-m \mid 00\rangle^{2}=-\frac{1}{3}
$$

which leads to $\xi^{\text {st }}\left(J_{\|}=0\right)=1 / \ln 3$. Fig.10 suggests the existence of 3 different regimes. In the weak coupling regime where $\xi^{\text {st }}>\xi$ the ladder can be effectively considered as a collection of weakly interacting closed spin 1 chains. In the strong coupling regime where $\xi^{\text {st }}>\xi$ the bonds are meanly along the rungs and the interbond coupling is small. Finally there is an intermediate region, with $\xi^{\text {st }}<\xi$, where the islands of spins interact strongly with their neighbours.

\section{The $A A_{3 / 2}$-ladder}

In table 6 we give the GS energy densities and spin correlation lengths of the ladder $A A_{3 / 2}$. As one may expect the correlation length is longer than for the spin 1 and $1 / 2$ ladders. This fact agrees with the results obtained by mapping the spin ladders into the NLSM [16 18].

\begin{tabular}{ccc}
\hline$J_{\|} / J_{\perp}$ & $-e_{\infty} / 2 J_{\perp}$ & $\xi$ \\
\hline \hline 0.0 & 1.875000 & 0.0000 \\
\hline 0.2 & 2.054760 & 1.8760 \\
\hline 0.4 & 2.449827 & 3.3099 \\
\hline 0.6 & 2.911353 & 3.9475 \\
\hline 0.8 & 3.400562 & 4.2401 \\
\hline 1.0 & 3.904988 & 4.3829 \\
\hline 1.25 & 4.548607 & 4.4624 \\
\hline 1.66 & 5.623131 & 4.4900 \\
\hline
\end{tabular}

Table 6. GS energy per site and correlation length of the $A A_{3 / 2}$ ladder.

\section{AKLT states for ladders}

The spin 3/2 2-ladder offers the possibility of constructing an AKLT state with a valence bond connecting every spin $3 / 2$ to its three nearest neighbours. More generally, let us consider a ladder with spin $S \geq 3 / 2$ and three integers $p, q, r \geq 1$ satisfying the eq. $2 S=p+q+r$. Then one can define an AKLT state, denoted by the triplet $(p, q, r)$, by linking the $2 S$ "elementary spinors" of each spin to the ones in its neighbours following the pattern shown in fig.(12). The AKLT states $(p, q, r)$ and $(q, p, r)$ when $p \neq q$ correspond to dimerized ladders and they differ by the translation of one unit space along the legs.

The spin 3/2 AKLT ladder corresponds in the above notation to $(1,1,1)$. This state contains in fact a spin 0 and a spin 1 state which can be generated by the MP equation (35) where the amplitudes $A_{J_{1} J_{2}}^{\lambda}$ are given by 9 -j symbols,

$$
A_{J_{1} J_{2}}^{\lambda}=3 \sqrt{\left(2 J_{2}+1\right)(2 \lambda+1)}\left\{\begin{array}{ccc}
1 / 2 & 1 / 2 & J_{2} \\
3 / 2 & 3 / 2 & \lambda \\
1 & 1 & J_{1}
\end{array}\right\}
$$


In this eq. $J_{1}, J_{2}=0$ and 1 , while $\lambda=0,1,2$.

The proof of (77) follows from the definition of the $9-\mathrm{j}$ symbols as the coefficients that give the change of basis when coupling in two different ways 4 angular momenta, namely [30]

$$
\begin{gathered}
\psi\left(j_{1} j_{3}\left(J_{13}\right) j_{2} j_{4}\left(J_{24}\right) J\right) \\
=\sum_{J_{12} J_{34}} \sqrt{\left(2 J_{12}+1\right)\left(2 J_{34}+1\right)\left(2 J_{13}+1\right)\left(2 J_{24}+1\right)} \\
\times\left\{\begin{array}{ccc}
j_{1} & j_{2} & J_{12} \\
j_{3} & j_{4} & J_{34} \\
J_{13} & J_{24} & J
\end{array}\right\} \psi\left(j_{1} j_{2}\left(J_{12}\right) j_{3} j_{4}\left(J_{34}\right) J\right)
\end{gathered}
$$

where $\psi\left(j_{1} j_{3}\left(J_{13}\right) j_{2} j_{4}\left(J_{24}\right) J\right)$ is a state with angular momentum $J$ obtained by the tensor product decomposition $J_{13} \otimes J_{24} \rightarrow J$, which in turn are obtained by the decompositions $j_{1} \otimes j_{3} \rightarrow J_{13}$ and $j_{2} \otimes j_{4} \rightarrow J_{24}$.

One may check that the normalization conditions (39) holds for (77), as a consequence of the orthogonality conditions satisfied by the 9 -j symbols [30]. The GS energy per site and the spin correlation length of the AKLT state (77) in the case where $J_{\|}=J_{\perp}=J$ are given by,

$$
e_{\infty}^{\mathrm{AKLT}} / 2 J=-3.263536, \quad \xi^{\mathrm{AKLT}}=1.116221
$$

This state has a much shorter correlation length than the MP state that minimizes the GS energy of the $A A_{3 / 2^{-}}$ ladder (see table 6 ). The GS energies of both states are also quite different. We conclude from these facts that the spin $3 / 2$ AKLT state does not give a good description of the GS of the $A A_{3 / 2}$-ladder.

A generic AKLT state of the type $(p, q, r)$ when $p \neq q$ has to be described by alternating MP amplitudes depending on the eveness of the site. Thus for even sites one has

$A_{J_{1} J_{2}}^{\lambda}=(q+r+1) \sqrt{\left(2 J_{2}+1\right)(2 \lambda+1)}\left\{\begin{array}{ccc}\frac{p}{2} & \frac{p}{2} & J_{2} \\ S & S & \lambda \\ \frac{q+r}{2} & \frac{q+r}{2} & J_{1}\end{array}\right\}$

where $J_{1}=0, \ldots, q ; J_{2}=0, \ldots, p$ and $\lambda=0, \ldots, 2 S-$ $r$. For odd sites the corresponding MP amplitudes are obtained by interchanging $p$ and $q$ in (80).

\section{V) CONCLUSIONS AND PROSPECTS}

Let us summarize the main results obtained in this paper.

- We have presented a rotational invariant formulation of the MPM which allow us to express the GS energy density, the correlation length and the string order parameter, in terms of invariant objects. This reduces considerably the number of independent MP parameters used in the minimization process.
- We have improved the numerical results concerning the GS energy density and spin correlation length obtained previously with other approximate methods as those of references [24,23. The consideration of MP ansatzs with multiple states per spin will certainly lead to better results.

- We have shown the equivalence between the ladder $A F_{1 / 2}$ and the spin 1 antiferromagnetic Heisenberg chain. The MPM applied to both systems shows strong numerical coincidences for the GS energy and correlation length. This agrees with the results obtained previously by other methods [26 28]

- We have found numerical evidences for the duality properties proposed in 29] for the spin ladders with magnetic structures $A A, A F$ and $F A$.

- We have shown that there is a breaking of the long range topological order of the spin 1 chains when they are coupled in a 2 legged ladder. A physical picture of the GS of the spin 1 ladder is given in terms of resonating closed spin 1 chains.

- We have constructed AKLT states for 2 legged ladders with spin $S \geq 3 / 2$, showing that the corresponding MP parameters are given by $9-\mathrm{j}$ symbols.

- We have suggested a relation between the MPM and the DMRG based on the density matrix that appear in both methods (see also [7]). We conjecture that the minimization of the GS energy $e_{\infty}$ can be transformed into an eigenvalue problem on a superblock $B_{N} \bullet B_{N}^{R}$.

In summary we have shown the adequacy of the MPM to study the 2 legged ladder, specially in the strong and intermediate coupling regimes. This is made possible from the fact that these ladders are finitely correlated. Hence one may expect that even spin ladders with a finite number of legs could be described by the same technique, although with a larger number of states $m$. On the other hand odd legged ladders are not finitely correlated and they cannot be properly described in the large $N$ limit within the actual formulation of the MPM. An interesting problem is the application of the MPM to 2D systems, which can be thougth of as ladders with a large number of legs. It is clear that one should choose a collection of the most representative states for the rungs to be added after each iteration of the MP recurrence equation.

Acknowledgements: JMR acknowledges financial support from a Basque Government FPI grant as well as from CICYT, contract AEN95-0590, and CIRIT, contract GRQ93-1047. Also he thanks G. Sierra, J. Dukelsky and M.A. Martín-Delgado for hospitality at the IMAFF (CSIC), Madrid, where this work was performed. GS and MAMD acknowledges support from the DIGICYT under 
contract No. PB96/0906 and JD acknowledges support from the DIGICYT under contract No. PB95/0123.

[1] K. G. Wilson, Rev. Mod. Phys. 47, 773 (1975).

[2] S. R. White, Phys. Rev. Lett. 69, 2863 (1992); Phys. Rev. B 48, 10345 (1993).

[3] I. Affleck, T. Kennedy, E.H. Lieb and H. Tasaki, Commun. Math. Phys. 115, 477 (1988).

[4] A. Klümper, A. Schadschneider and J. Zittartz, Europhys. Lett. 24, 293 (1993).

[5] S. Ostlund and S. Rommer, Phys. Rev. Lett. 75, 3537 (1995); S. Rommer and S. Ostlund, Phys. Rev. B 55, 2164 (1997).

[6] M. Fannes, B. Nachtergaele and R.F. Werner, Commun. Math. Phys. 144, 443 (1992) ( see references therein on the MPM).

[7] J. Dukelsky, M.A. Martín-Delgado, T. Nishino and G. Sierra, cond-mat/9710310.

[8] S. Brehmer, H.-J. Mikeska and U. Neugebauer, J. Phys. Condens. Matter 8, 7161 (1996).

[9] E. Dagotto and T. M. Rice, Science 271, 618 (1996).

[10] M. den Nijs and K. Rommelse, Phys. Rev. B 40, 4709 (1989).

[11] S.R. White and D.A. Huse, Phys. Rev. B 48, 3844 (1993).

[12] T. Nishino and K. Okunishi, J. Phys. Soc. Jpn.. 65, 891 (1996).

[13] H.J. Schulz, Phys. Rev. B 34, 6372 (1986).

[14] S. P. Strong and A. J. Millis, Phys. Rev. Lett. 69, 2419 (1992).

[15] D. G. Shelton, A. A. Nersesyan and A. M. Tsvelik, Phys. Rev. B 53, 8521 (1996).

[16] D. Senechal, Phys. Rev. B 52, 15319 (1995).

[17] G. Sierra, J. Phys. A 29, 3299 (1996).

[18] S. Dell'Aringa, E. Ercolessi, G. Morandi, P. Pieri and M. Roncaglia, Phys. Rev. Lett. 78, 2457 (1997).

[19] E. Dagotto, J. Riera and D. J. Scalapino, Phys. Rev. B 45, 5744 (1992).

[20] S. R. White, R. M. Noack and D. J. Scalapino, Phys. Rev. Lett. 73, 886 (1994).

[21] B. Frischmuth, B. Ammon and M. Troyer, Phys. Rev. B 54, R3714 (1996).

[22] M. Greven, R.J. Birgeneau and U.-J. Wiese, Phys. Rev. Lett. 77, 1865 (1996).

[23] S. Gopalan, T. M. Rice and M. Sigrist, Phys. Rev. B 49, 8901 (1994) and corrected version by B. Normand and T. M. Rice cond-mat/9701202.

[24] G. Sierra, M.A. Martín-Delgado, Phys. Rev. B 56, 8774 (1997).

[25] T. Barnes, E. Dagotto, J. Riera and E. S. Swanson, Phys. Rev. B 47, 3196 (1993).

[26] K. Hida, J. Phys. SOc. Jpn. 60, 1347 (1991).

[27] S. R. White, Phys. Rev. B 53, 52 (1996).

[28] H. Watanabe, Phys. Rev. B 52, 12508 (1995).

[29] G. Sierra, M.A. Martín-Delgado, cond-mat/9706104, to appear in J. Phys. A.
[30] D. A. Varshalovich, A. N. Moskalev and V. K. Khersonskii, "Quantum Theory of Angular Momenta", World Scientific (1988).

[31] Encyclopedic Dictionary of Mathematics, edited by S. Iyanaga and Y. Kawada (MIT Press, London, 1977).

\section{APPENDIX A: THE MP ANSATZ AND THE GRASSMANNIAN MANIFOLDS}

In this appendix we shall give a proof of eq.(位) which gives a precise mathematical meaning of the coefficients $A_{\alpha \beta}[s]$ defining a generic MP ansatz.

In the r.h.s. of eq.(1) we have a generic vector of dimension $n=m m^{*}$ while on its l.h.s. the vector has dimension $m$. Hence eq. (1) amounts to a choice of a $m$-dimensional linear subspace of $\mathbf{R}^{n}$ in the case of $A_{\alpha \beta}[s]$ real or a complex subspace of $\mathbf{C}^{n}$ in the case of $A_{\alpha \beta}[s]$ complex. Let us call the set of all these subspaces as $M_{n, m}(\mathbf{R})$ and $M_{n, m}(\mathbf{C})$ for $A_{\alpha \beta}[s]$ real and complex respectively. The group $O(n)$ ( resp. $U(n))$ acts transitively on $M_{n, m}(\mathbf{R})$ ( resp. $\left.M_{n, m}(\mathbf{C})\right)$, which leads to the result [31]

$$
\begin{aligned}
& M_{n, m}(\mathbf{R})=\frac{O(n)}{O(m) \otimes O(n-m)} \\
& M_{n, m}(\mathbf{C})=\frac{U(n)}{U(m) \otimes U(n-m)}
\end{aligned}
$$

In (81) the groups $O(m)$ and $O(n-m)$ are identified with the subgroups of $O(n)$ consisting of those elements leaving fixed every vector of a given $(n-m)$-dimensional subspace and of its orthogonal complement, respectively. Similar arguments lead to eq. (82). $M_{n, m}(\mathbf{R})\left(M_{n, m}(\mathbf{C})\right)$ are called the real (complex) Grassmannian manifolds. Taking $n=m m^{*}$ in (81) we get eq.(蛋).

As a simple illustration of these eqs. let us consider the case of a MP ansatz that generates a single state $|G S\rangle_{N}$ $(m=1)$, i.e.

$$
|G S\rangle_{N}=\sum_{s} A[s]|s\rangle_{N} \otimes|G S\rangle_{N-1}
$$

with $A[s] \in \mathbf{R}$. The normalization condition (2) reads,

$$
\sum_{s=1}^{m^{*}} A[s]^{2}=1
$$

Thus $A[s]$ belongs to the $\left(m^{*}-1\right)$-dimensional sphere $S O\left(m^{*}\right) / S O\left(m^{*}-1\right)$. Upon the identification of $A[s]$ and $-A[s]$ we get the $\left(m^{*}-1\right)$-real proyective space $M_{m^{*}, 1}(\mathbf{R})=S O\left(m^{*}\right) / S O\left(m^{*}-1\right) \otimes \mathbf{Z}_{2}$. 


\section{APPENDIX B: THE ROTATIONAL INVARIANT MPM}

\section{Group theoretical preliminaries}

Before we give the proof of the main formulas of section III we shall review some basic definitions and results in group theory [30].

An irreducible tensor with angular momentum $k$ is an operator $T_{M}^{(k)}(M=k, \cdots,-k)$ which satisfies the following commutation relations with the total angular momentum operator $\mathbf{J}$,

$$
\begin{gathered}
{\left[J_{z}, T_{M}^{(k)}\right]=M T_{M}^{(k)}} \\
{\left[J_{x} \pm i J_{y}, T_{M}^{(k)}\right]=\sqrt{k(k+1)-M(M \pm 1)} T_{M \pm 1}^{(k)}}
\end{gathered}
$$

The scalar product of two irreducible tensors $\mathbf{T}^{(k)}$ and $\mathbf{U}^{(k)}$ with the same spin $k$ is defined by,

$$
\mathbf{T}^{(k)} \cdot \mathbf{U}^{(k)}=\sum_{M=-k}^{k}(-1)^{-M} T_{M}^{(k)} U_{-M}^{(k)}
$$

The Wigner-Eckart theorem reads,

$$
\begin{gathered}
\left\langle J M\left|T_{\mu}^{(k)}\right| J^{\prime} M^{\prime}\right\rangle \\
=(-1)^{J-M}\left(\begin{array}{ccc}
J & k & J^{\prime} \\
-M & \mu & M^{\prime}
\end{array}\right)\left(J\left\|\mathbf{T}^{(k)}\right\| J^{\prime}\right)
\end{gathered}
$$

where the 3 -j symbol is related to the CG coefficient by

$$
\left(\begin{array}{ccc}
J & k & J^{\prime} \\
-M & \mu & M^{\prime}
\end{array}\right)=\frac{(-1)^{J-k-M^{\prime}}}{\sqrt{2 J^{\prime}+1}}\left\langle J-M k \mu \mid J^{\prime}-M^{\prime}\right\rangle
$$

The quantity $\left(J\left\|\mathbf{T}^{(k)}\right\| J^{\prime}\right)$ in (87) is called the reduced matrix element of the operator $\mathbf{T}^{(k)}$. As an example we give the reduced matrix element of the spin operator $\mathbf{S}$,

$$
(S|| \mathbf{S}|| S)=\sqrt{S(S+1)(2 S+1)}
$$

Let $\left|\alpha_{1} j_{1} \alpha_{2} j_{2} J M\right\rangle$ be a state with total angular momenta $J$ and third component $M$, appearing in the tensor product decomposition $\left(\alpha_{1} j_{1}\right) \otimes\left(\alpha_{2} j_{2}\right)$, where $(\alpha j)$ denotes a state with total angular momentum $j$ and $\alpha$ labels other possible quantum numbers. We shall need below the following results.

$$
\begin{gathered}
\left\langle\alpha_{1} j_{1} \alpha_{2} j_{2} J M\left|\left(\mathbf{T}_{1}^{(k)} \cdot \mathbf{T}_{2}^{(k)}\right)\right| \alpha_{1}^{\prime} j_{1}^{\prime} \alpha_{2}^{\prime} j_{2}^{\prime} J^{\prime} M^{\prime}\right\rangle \\
=\delta_{J J^{\prime}} \delta_{M M^{\prime}}(-1)^{j_{2}+J+j_{1}^{\prime}}\left\{\begin{array}{ccc}
j_{1} & j_{2} & J \\
j_{2}^{\prime} & j_{1}^{\prime} & k
\end{array}\right\} \\
\times\left(\alpha_{1} j_{1}\left\|\mathbf{T}_{1}^{(k)}\right\| \alpha_{1}^{\prime} j_{1}^{\prime}\right)\left(\alpha_{2} j_{2}\left\|\mathbf{T}_{2}^{(k)}\right\| \alpha_{2}^{\prime} j_{2}^{\prime}\right) \\
\left(\alpha_{1} j_{1} \alpha_{2} j_{2} J\left\|\mathbf{T}_{1}^{(k)}\right\| \alpha_{1}^{\prime} j_{1}^{\prime} \alpha_{2}^{\prime} j_{2}^{\prime} J^{\prime}\right) \\
=\delta_{\alpha_{2} \alpha_{2}^{\prime}} \delta_{j_{2} j_{2}^{\prime}}(-1)^{j_{1}+j_{2}+J^{\prime}+k}\left\{\begin{array}{ccc}
j_{1} & J & j_{2} \\
J^{\prime} & j_{1}^{\prime} & k
\end{array}\right\} \\
\times \sqrt{(2 J+1)\left(2 J^{\prime}+1\right)}\left(\alpha_{1} j_{1}\left\|\mathbf{T}_{1}^{(k)}\right\| \alpha_{1}^{\prime} j_{1}^{\prime}\right)
\end{gathered}
$$

$$
\begin{gathered}
\left(\alpha_{1} j_{1} \alpha_{2} j_{2} J\left\|\mathbf{T}_{2}^{(k)}\right\| \alpha_{1}^{\prime} j_{1}^{\prime} \alpha_{2}^{\prime} j_{2}^{\prime} J^{\prime}\right) \\
=\delta_{\alpha_{1} \alpha_{1}^{\prime}} \delta_{j_{1} j_{1}^{\prime}}(-1)^{j_{1}+j_{2}^{\prime}+J+k}\left\{\begin{array}{ccc}
j_{2} & J & j_{1} \\
J^{\prime} & j_{2}^{\prime} & k
\end{array}\right\} \\
\times \sqrt{(2 J+1)\left(2 J^{\prime}+1\right)}\left(\alpha_{2} j_{2}\left\|\mathbf{T}_{2}^{(k)}\right\| \alpha_{2}^{\prime} j_{2}^{\prime}\right)
\end{gathered}
$$

The subindices 1 and 2 in $\mathbf{T}_{1}^{(k)}$ and $\mathbf{T}_{2}^{(k)}$ mean that the corresponding operators acts on the states labelled as $\left(\alpha_{1} j_{1}\right)$ and $\left(\alpha_{2} j_{2}\right)$ respectively.

Recursion relations for the scalar product of invariant tensors

We want to prove eq.(411).

Using eq.(35) we easily get for $N>n>m$,

$$
{ }_{N}\left\langle J_{1} M\left|\mathcal{O}^{(k, A)}(n) \cdot \mathcal{O}^{(k, B)}(m)\right| J_{1} M\right\rangle_{N}
$$

$=\sum_{J_{2}} T_{J_{1}, J_{2}}{ }_{N-1}\left\langle J_{2} M\left|\mathcal{O}^{(k, A)}(n) \cdot \mathcal{O}^{(k, B)}(m)\right| J_{2} M\right\rangle_{N-1}$

where $T_{J_{1}, J_{2}}$ is given in (42). Iterating (93) $N-n$ times we reach the situation where $N=n$. This produces the term $T_{J_{1}, J_{2}}^{N-n}$ in (41). Next we need to compute the matrix element,

$$
\begin{gathered}
{ }_{n}\left\langle J_{1} M\left|\mathcal{O}^{(k, A)}(n) \cdot \mathcal{O}^{(k, B)}(m)\right| J_{1} M\right\rangle_{n} \\
=\sum_{J_{2} J_{3} \lambda_{2} \lambda_{3}}\left(A_{J_{1} J_{2}}^{\lambda_{2}}\right)^{*} A_{J_{1} J_{3}}^{\lambda_{3}} \\
{ }_{n}\left\langle\left(\lambda_{2} J_{2}\right), J_{1} M\left|\mathcal{O}^{(k, A)}(n) \cdot \mathcal{O}^{(k, B)}(m)\right|\left(\lambda_{3} J_{3}\right), J_{1} M\right\rangle_{n}
\end{gathered}
$$

The matrix element on the r.h.s. of (94) has the form described in (90), which yields,

$$
\begin{gathered}
{ }_{n}\left\langle\left(\lambda_{2} J_{2}\right), J_{1} M\left|\mathcal{O}^{(k, A)}(n) \cdot \mathcal{O}^{(k, B)}(m)\right|\left(\lambda_{3} J_{3}\right), J_{1} M\right\rangle_{n} \\
=(-1)^{J_{1}+J_{2}+\lambda_{3}}\left\{\begin{array}{ccc}
\lambda_{2} & J_{2} & J_{1} \\
J_{3} & \lambda_{3} & k
\end{array}\right\} \\
\times{ }_{n}\left(\lambda_{2}\left\|\mathcal{O}^{(k, A)}(n)\right\| \lambda_{3}\right)_{n}{ }_{n-1}\left(J_{2}\left\|\mathcal{O}^{(k, B)}(m)\right\| J_{3}\right)_{n-1}
\end{gathered}
$$

Introducing (95) into (94) we find

$$
\begin{aligned}
& { }_{n}\left\langle J_{1} M\left|\mathcal{O}^{(k, A)}(n) \cdot \mathcal{O}^{(k, B)}(m)\right| J_{1} M\right\rangle_{n} \\
& =\sum_{J_{2} J_{3}} \widehat{\mathcal{O}}_{J_{1}, J_{2} J_{3} n-1}^{(k, A)}\left(J_{2}\left\|\mathcal{O}^{(k, B)}(m)\right\| J_{3}\right)_{n-1}
\end{aligned}
$$

where

$$
\begin{gathered}
\widehat{\mathcal{O}}_{J_{1}, J_{2} J_{3}}^{(k, A)}=\sum_{\lambda_{2}, \lambda_{3}}\left(A_{J_{1} J_{2}}^{\lambda_{2}}\right)^{*} A_{J_{1} J_{3}}^{\lambda_{3}} \\
\times(-1)^{\lambda_{3}+J_{1}+J_{2}}\left\{\begin{array}{ccc}
\lambda_{2} & J_{2} & J_{1} \\
J_{3} & \lambda_{3} & k
\end{array}\right\}\left(\lambda_{2}\left\|\mathcal{O}^{(k, A)}\right\| \lambda_{3}\right)
\end{gathered}
$$

The next step is to apply the MP ansatz (35) to

$$
{ }_{n}\left(J_{1}\left\|\mathcal{O}^{(k, B)}(m)\right\| J_{2}\right)_{n}
$$




$$
\begin{gathered}
=\sum_{\lambda_{1} \lambda_{2}}\left(A_{J_{1} J_{3}}^{\lambda_{1}}\right)^{*} A_{J_{2} J_{4}}^{\lambda_{2}} \\
\times_{n}\left(\left(\lambda_{1} J_{3}\right), J_{1}\left\|\mathcal{O}^{(k, B)}(m)\right\|\left(\lambda_{2} J_{4}\right), J_{2}\right)_{n}
\end{gathered}
$$

For $n>m$ we can use (92), getting

$$
\begin{aligned}
& { }_{n}\left(\left(\lambda_{1} J_{3}\right), J_{1}\left\|\mathcal{O}^{(k, B)}(m)\right\|\left(\lambda_{2} J_{4}\right), J_{2}\right)_{n} \\
= & \delta_{\lambda_{1} \lambda_{2}}(-1)^{\lambda_{1}+J_{1}+J_{4}+k} \sqrt{\left(2 J_{1}+1\right)\left(2 J_{2}+1\right)} \\
\times & \left\{\begin{array}{ccc}
J_{3} & J_{1} & \lambda_{1} \\
J_{2} & J_{4} & k
\end{array}\right\}{ }_{n-1}\left(J_{3}\left\|\mathcal{O}^{(k, B)}(m)\right\| J_{4}\right)_{n-1}
\end{aligned}
$$

Plugging (99) into (98) we get,

$$
\begin{gathered}
{ }_{n}\left(J_{1}\left\|\mathcal{O}^{(k, B)}(m)\right\| J_{2}\right)_{n} \\
=\sum_{J_{3} J_{4}}\left(T_{k}\right)_{J_{1} J_{2}, J_{3} J_{4}} n-1\left(J_{3}\left\|\mathcal{O}^{(k, B)}(m)\right\| J_{4}\right)_{n-1}, \quad(n>m)
\end{gathered}
$$

where $\left(T_{k}\right)_{J_{1} J_{2}, J_{3} J_{4}}$ is defined in 43 . The term $T_{k}^{n-m-1}$ in (41) results from the iteration of (100) until one gets $n=m$. In the case when $n=m$ in (99) we should apply (91) obtaining

$$
\begin{aligned}
& { }_{n}\left(\left(\lambda_{1} J_{3}\right), J_{1}\left\|\mathcal{O}^{(k, B)}(n)\right\|\left(\lambda_{2} J_{4}\right), J_{2}\right)_{n} \\
= & \delta_{J_{3} J_{4}}(-1)^{\lambda_{1}+J_{2}+J_{3}+k} \sqrt{\left(2 J_{1}+1\right)\left(2 J_{2}+1\right)} \\
& \times\left\{\begin{array}{ccc}
\lambda_{1} & J_{1} & J_{3} \\
J_{2} & \lambda_{2} & k
\end{array}\right\}{ }_{n}\left(\lambda_{1}\left\|\mathcal{O}^{(k, B)}(n)\right\| \lambda_{2}\right)_{n}
\end{aligned}
$$

Introducing (101) into (98) we get,

$$
{ }_{n}\left(J_{1}\left\|\mathcal{O}^{(k, B)}(n)\right\| J_{2}\right)_{n}=\sum_{J_{3}} \widehat{\mathcal{O}}_{J_{1} J_{2}, J_{3}}^{(k, B)}
$$

where

$$
\begin{gathered}
\widehat{\mathcal{O}}_{J_{1} J_{2}, J_{3}}^{(k, B)}=\sum_{\lambda_{1} \lambda_{2}}\left(A_{J_{1} J_{3}}^{\lambda_{1}}\right)^{*} A_{J_{2} J_{3}}^{\lambda_{2}}(-1)^{\lambda_{1}+J_{2}+J_{3}+k} \\
\times \sqrt{\left(2 J_{1}+1\right)\left(2 J_{2}+1\right)}\left\{\begin{array}{lll}
\lambda_{1} & J_{1} & J_{3} \\
J_{2} & \lambda_{2} & k
\end{array}\right\}\left(\lambda_{1}\left\|\mathcal{O}^{(k, B)}\right\| \lambda_{2}\right)
\end{gathered}
$$

This ends the proof of eq.(41).

Recursion relation of the energy expectation values

We shall not give here the explicit proof of eq.(50) since it is quite analogous to the one performed in the previous paragraph. We shall simply state the result.

The matrix $\hat{h}_{J_{1}, J_{2}}$ appearing in (50) is given by the sum

$$
\hat{h}_{J_{1}, J_{2}}=\hat{h}_{J_{1}, J_{2}}^{(1)}+\hat{h}_{J_{1}, J_{2}}^{(2)}
$$

where

$$
\widehat{h}_{J_{1}, J_{2}}^{(1)}=J_{\perp} \sum_{\lambda}\left(\frac{1}{2} \lambda(\lambda+1)-S(S+1)\right)\left|A_{J_{1} J_{2}}^{\lambda}\right|^{2}
$$

$$
\begin{aligned}
\widehat{h}_{J_{1}, J_{4}}^{(2)}= & 2 J_{\|} \sum_{J_{2} J_{3} J_{4}, \lambda_{1}, \ldots, \lambda_{4}}\left(A_{J_{1} J_{2}}^{\lambda_{1}} A_{J_{2} J_{4}}^{\lambda_{3}}\right)^{*} A_{J_{1} J_{3}}^{\lambda_{2}} A_{J_{3} J_{4}}^{\lambda_{4}} \\
& \times(-1)^{1+\lambda_{3}+\lambda_{4}} \xi_{J_{2} J_{3} J_{1}}^{\lambda_{2} \lambda_{1}} \xi_{J_{3} J_{2} J_{4}}^{\lambda_{3} \lambda_{4}}
\end{aligned}
$$

and

$$
\begin{aligned}
& \xi_{J_{1} J_{2} J_{3}}^{\lambda_{1} \lambda_{2}}=(-1)^{J_{1}+J_{3}} \sqrt{\left(2 J_{1}+1\right)\left(2 \lambda_{1}+1\right)\left(2 \lambda_{2}+1\right)} \\
& \times \sqrt{S(S+1)(2 S+1)}\left\{\begin{array}{lll}
\lambda_{1} & \lambda_{2} & 1 \\
J_{1} & J_{2} & J_{3}
\end{array}\right\}\left\{\begin{array}{ccc}
\lambda_{1} & \lambda_{2} & 1 \\
S & S & S
\end{array}\right\}
\end{aligned}
$$

where the following property for the 6 -j symbol with an element equal 1 has been used [30]:

$$
\left\{\begin{array}{lll}
\lambda_{1} & \lambda_{2} & 1 \\
J_{1} & J_{2} & J_{3}
\end{array}\right\}=\left\{\begin{array}{ccc}
\lambda_{2} & \lambda_{1} & 1 \\
J_{2} & J_{1} & J_{3}
\end{array}\right\}
$$

\section{APPENDIX C: THE STRING ORDER PARAMETER OF SPIN 1 CHAIN AND LADDER}

Let us first consider the spin 1 chain. The MP ansatz is given simply by,

$$
\left|J_{1} M_{1}\right\rangle_{N}=\sum_{J_{2}} A_{J_{1} J_{2}}\left|\left(1 J_{2}\right), J_{1} M_{1}\right\rangle_{N}
$$

where the state $\left|\left(1 J_{2}\right), J_{1} M_{1}\right\rangle_{N}$ reads as in (36) with $\lambda=1$. We shall choose half-integer values of the angular momenta $J_{1}$ and $J_{2}$ which amounts to have a spin $1 / 2$ at one end of the chain [5,7].

We shall next show that the operators $T=\widehat{1}$ and $\widehat{e^{i \pi S^{z}}}$ (103have both an eigenvalue equal to 1 . Let us first of all write out explicitely their components,

$$
\begin{gathered}
(T)_{J_{1} M_{1} J_{1}^{\prime} M_{1}^{\prime}, J_{2} M_{2} J_{2}^{\prime} M_{2}^{\prime}} \\
=\delta_{M_{1}-M_{2}, M_{1}^{\prime}-M_{2}^{\prime}} A_{J_{1} J_{2}} A_{J_{1}^{\prime} J_{2}^{\prime}} \\
\times\left\langle 1 M_{1}-M_{2}, J_{2} M_{2} \mid J_{1} M_{1}\right\rangle\left\langle 1 M_{1}^{\prime}-M_{2}^{\prime}, J_{2}^{\prime} M_{2}^{\prime} \mid J_{1}^{\prime} M_{1}^{\prime}\right\rangle \\
\left(\widehat{e^{i \pi S^{z}}}\right)_{J_{1} M_{1} J_{1}^{\prime} M_{1}^{\prime}, J_{2} M_{2} J_{2}^{\prime} M_{2}^{\prime}} \\
\times \delta_{M_{1}-M_{2}, M_{1}^{\prime}-M_{2}^{\prime}}(-1)^{M_{1}-M_{2}} A_{J_{1} J_{2}} A_{J_{1}^{\prime} J_{2}^{\prime}} \\
\times\left\langle 1 M_{1}-M_{2}, J_{2} M_{2} \mid J_{1} M_{1}\right\rangle\left\langle 1 M_{1}^{\prime}-M_{2}^{\prime}, J_{2}^{\prime} M_{2}^{\prime} \mid J_{1}^{\prime} M_{1}^{\prime}\right\rangle
\end{gathered}
$$

The normalization conditions on $A_{J_{1} J_{2}}$ read 


$$
\sum_{J_{2}} A_{J_{1} J_{2}}^{2}=1, \forall J_{1}
$$

Using these eqs. and the properties of the CG coefficients, one can verify that $v$ and $v^{\text {st }}$ defined as

$$
\begin{gathered}
v_{J_{1} M_{1} J_{1}^{\prime} M_{1}^{\prime}}=\delta_{J_{1} J_{1}^{\prime}} \delta_{M_{1} M_{1}^{\prime}} \\
v_{J_{1} M_{1} J_{1}^{\prime} M_{1}^{\prime}}^{\mathrm{st}}=\delta_{J_{1} J_{1}^{\prime}} \delta_{M_{1} M_{1}^{\prime}}(-1)^{M_{1}-1 / 2}
\end{gathered}
$$

are right eigenvectors with eigenvalue 1 of the matrices $T$ and $e^{\widehat{i \pi S^{z}}}$ respectively.

Similarly the left eigenvectors associated to this eigenvalue are given by,

$$
\begin{gathered}
\rho_{J_{1} M_{1} J_{1}^{\prime} M_{1}^{\prime}}=\delta_{J_{1} J_{1}^{\prime}} \delta_{M_{1} M_{1}^{\prime}} \rho_{J_{1}} /\left(2 J_{1}+1\right) \\
\rho_{J_{1} M_{1} J_{1}^{\prime} M_{1}^{\prime}}^{\mathrm{st}}=\delta_{J_{1} J_{1}^{\prime}} \delta_{M_{1} M_{1}^{\prime}}(-1)^{M_{1}-1 / 2} \rho_{J_{1}} /\left(2 J_{1}+1\right)
\end{gathered}
$$

where $\rho_{J}$ is the left eigenvector with eigenvalue 1 of the matrix $T_{J_{1} J_{2}}=A_{J_{1} J_{2}}^{2}$.

According to eq. (22) the string order parameter $g(\infty)$ is given by the product of two matrix elements which we compute below.

Let us first consider,

$$
\begin{gathered}
\left\langle\rho\left|\widehat{S^{z}}\right| v^{\mathrm{st}}\right\rangle \\
=\sum \rho_{J_{1} M_{1} J_{1}^{\prime} M_{1}^{\prime}}{\widehat{S^{z}}}_{J_{1} M_{1} J_{1}^{\prime} M_{1}^{\prime}, J_{2} M_{2} J_{2}^{\prime} M_{2}^{\prime}} v_{J_{2} M_{2} J_{2}^{\prime} M_{2}^{\prime}}
\end{gathered}
$$

The hated version of $S^{z}$ is given by,

$$
\begin{gathered}
\left(\widehat{S^{z}}\right)_{J_{1} M_{1} J_{1}^{\prime} M_{1}^{\prime}, J_{2} M_{2} J_{2}^{\prime} M_{2}^{\prime}} \\
=\delta_{M_{1}-M_{2}, M_{1}^{\prime}-M_{2}^{\prime}}\left(M_{1}-M_{2}\right) A_{J_{1} J_{2}} A_{J_{1}^{\prime} J_{2}^{\prime}} \\
\times\left\langle 1 M_{1}-M_{2}, J_{2} M_{2} \mid J_{1} M_{1}\right\rangle\left\langle 1 M_{1}^{\prime}-M_{2}^{\prime}, J_{2}^{\prime} M_{2}^{\prime} \mid J_{1}^{\prime} M_{1}^{\prime}\right\rangle
\end{gathered}
$$

which together with 112,113 ) lead to,

$$
\begin{gathered}
\left\langle\rho\left|\widehat{S^{z}}\right| v^{\text {st }}\right\rangle=\sum \frac{\rho_{J_{1}}}{2 J_{1}+1} A_{J_{1} J_{2}}^{2} \\
\times(-1)^{M_{2}-1 / 2}\left(M_{1}-M_{2}\right)\left(\left\langle 1 M_{1}-M_{2}, J_{2} M_{2} \mid J_{1} M_{1}\right\rangle\right)^{2}
\end{gathered}
$$

Similarly we get

$$
\begin{gathered}
\left\langle\rho^{\mathrm{st}}\left|\widehat{S^{z}}\right| v\right\rangle=\sum \frac{\rho_{J_{1}}}{2 J_{1}+1} A_{J_{1} J_{2}}^{2} \\
\times(-1)^{M_{1}-1 / 2}\left(M_{1}-M_{2}\right)\left(\left\langle 1 M_{1}-M_{2}, J_{2} M_{2} \mid J_{1} M_{1}\right\rangle\right)^{2}
\end{gathered}
$$

Observing that

$$
(-1)^{M_{1}-1 / 2}\left(M_{1}-M_{2}\right)=-(-1)^{M_{2}-1 / 2}\left(M_{1}-M_{2}\right)
$$

where $M_{1}-M_{2}=0, \pm 1$, we obtain

$$
\left\langle\rho^{\mathrm{st}}\left|\widehat{S^{z}}\right| v\right\rangle=-\left\langle\rho\left|\widehat{S^{z}}\right| v^{\mathrm{st}}\right\rangle
$$

which in turn implies

$$
g(\infty)=-\left(\left\langle\rho\left|\widehat{S^{z}}\right| v^{\mathrm{st}}\right\rangle\right)^{2}
$$

Let us come back to eq. (115), which can be written as

$\sum \frac{-\rho_{J_{1}}}{2 J_{1}+1} A_{J_{1} J_{2}}^{2}(-1)^{M_{1}-1 / 2}\left\langle\left(1 J_{2}\right) J_{1} M_{1}\left|S_{1}^{z}\right|\left(1 J_{2}\right) J_{1} M_{1}\right\rangle$

where $S_{1}^{z}$ denotes the spin operator acting on the spin 1 . Using the Wigner-Eckart theorem we get,

$$
\begin{gathered}
\left\langle\left(1 J_{2}\right) J_{1} M_{1}\left|S_{1}^{z}\right|\left(1 J_{2}\right) J_{1} M_{1}\right\rangle \\
=(-1)^{J_{1}-M_{1}}\left(\begin{array}{ccc}
J_{1} & 1 & J_{1} \\
-M_{1} & 0 & M_{1}
\end{array}\right)\left(\left(1 J_{2}\right) J_{1}\left\|\mathbf{S}_{1}\right\|\left(1 J_{2}\right) J_{1}\right) \\
=\frac{M_{1}}{\sqrt{J_{1}\left(2 J_{1}+1\right)\left(J_{1}+1\right)}}\left(\left(1 J_{2}\right) J_{1}\left\|\mathbf{S}_{1}\right\|\left(1 J_{2}\right) J_{1}\right)
\end{gathered}
$$

The reduced matrix element appearing in 121 can be computed using (91),

$$
\begin{aligned}
& \left(\left(1 J_{2}\right) J_{1}\left\|\mathbf{S}_{1}\right\|\left(1 J_{2}\right) J_{1}\right) \\
& =\sqrt{6}(-1)^{J_{1}+J_{2}}\left(2 J_{1}+1\right)\left\{\begin{array}{ccc}
1 & J_{1} & J_{2} \\
J_{1} & 1 & 1
\end{array}\right\} \\
& =\frac{\sqrt{2 J_{1}+1}\left(2+J_{1}\left(J_{1}+1\right)-J_{2}\left(J_{2}+1\right)\right)}{2 \sqrt{J_{1}\left(J_{1}+1\right)}}
\end{aligned}
$$

Substituting (121.122) into (120) and performing the sum over $M_{1}$ with the aid of the formula,

$$
\sum_{M=-J}^{J} M(-1)^{M-\frac{1}{2}}=\left(J+\frac{1}{2}\right)(-1)^{J-1 / 2},(J \text { : half integer })
$$

we get finally,

$$
\begin{aligned}
& \left\langle\rho\left|\widehat{S^{z}}\right| v^{\mathrm{st}}\right\rangle=\frac{1}{4} \sum \rho_{J_{1}} A_{J_{1} J_{2}}^{2}(-1)^{J_{1}-1 / 2} \\
& \times \frac{2+J_{1}\left(J_{1}+1\right)-J_{2}\left(J_{2}+1\right)}{J_{1}\left(J_{1}+1\right)}
\end{aligned}
$$

From eqs 119 124) we immediately get the value of $g(\infty)$ in the AKLT case,

$$
\mathrm{AKLT}: A_{\frac{1}{2} \frac{1}{2}}=1 \rightarrow g(\infty)=-(2 / 3)^{2}
$$

In ref [7] the spin 1 Heisenberg chain was studied with a MP ansatz built up with two states with $J=1 / 2$ and $3 / 2$. The values of the MP parameters obtained in [7] are reproduced below

$$
\begin{gathered}
A_{\frac{1}{2} \frac{1}{2}}=0.988995, A_{\frac{1}{2} \frac{3}{2}}=0.14795 \\
A_{\frac{3}{2} \frac{1}{2}}=-0.952887, A_{\frac{3}{2} \frac{3}{2}}=-0.303325 \\
\rho_{\frac{1}{2}}=0.97646, \rho_{\frac{3}{2}}=0.023539
\end{gathered}
$$

Introducing (126) into eqs.(119) and (124) we get $g(\infty)=-0.387$, which can be compared with the exact value given by -0.374325 [11]. In [5] the spin 1 chain 
was studied with a MP ansatz with two spin $1 / 2$ and two spin $3 / 2$ states, which yields $g(\infty)=-0.3759$. This shows again that MP ansatzs with multiplicity improve considerably the accuracy of the numerical results [5,7].

Let us go now to the spin 1 ladder. In section IV we gave an intuitive argument which suggested that the LRTO of the single spin 1 chains is destroyed by the interchain coupling. Next we show that this is indeed what happens.

Let us first write eqs (108,109) in the case of ladders.

$$
\begin{aligned}
& (T)_{J_{1} M_{1} J_{1}^{\prime} M_{1}^{\prime}, J_{2} M_{2} J_{2}^{\prime} M_{2}^{\prime}}
\end{aligned}
$$

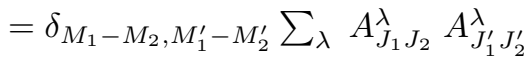

$$
\begin{aligned}
& \times\left\langle\lambda M_{1}-M_{2}, J_{2} M_{2} \mid J_{1} M_{1}\right\rangle\left\langle\lambda M_{1}^{\prime}-M_{2}^{\prime}, J_{2}^{\prime} M_{2}^{\prime} \mid J_{1}^{\prime} M_{1}^{\prime}\right\rangle \\
& \left(e^{\widehat{i \pi S_{1}^{z}}}\right)_{J_{1} M_{1} J_{1}^{\prime} M_{1}^{\prime}, J_{2} M_{2} J_{2}^{\prime} M_{2}^{\prime}} \\
& =\delta_{M_{1}-M_{2}, M_{1}^{\prime}-M_{2}^{\prime}} \sum_{\lambda \lambda^{\prime}} A_{J_{1} J_{2}}^{\lambda} A_{J_{1}^{\prime} J_{2}^{\prime}}^{\lambda^{\prime}} \\
& \times\left\langle 1 M_{1}-M_{2}, J_{2} M_{2} \mid J_{1} M_{1}\right\rangle\left\langle 1 M_{1}^{\prime}-M_{2}^{\prime}, J_{2}^{\prime} M_{2}^{\prime} \mid J_{1}^{\prime} M_{1}^{\prime}\right\rangle \\
& \times\left\langle\lambda M_{1}-M_{2}\left|e^{i \pi S_{1}^{z}}\right| \lambda^{\prime} M_{1} M_{2}\right\rangle
\end{aligned}
$$

where $S_{1}^{z}$ denotes the spin operator acting on the first

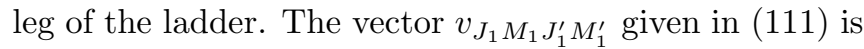
an eigenvector with eigenvalue 1 of the matrix $T$ defined by (127). This property is a consequence of the normalization condition (39). For the spin 1 chain the latter condition also guarantees the existence of an eigenvalue 1 of the operator (109). However this is not generally the case for the operator $(128)$.

The last matrix element in (128) can be deduced expressing the state $|\lambda \mu\rangle$ of the rung in terms of the spin 1 states of every site,

$$
|\lambda \mu\rangle=\sum_{m_{1} m_{2}}\left|1 m_{1}\right\rangle_{1}\left|s m_{2}\right\rangle_{2}\left\langle 1 m_{1} 1 m_{2} \mid \lambda \mu\right\rangle
$$

We thus get

$$
\begin{aligned}
& \left(e^{\widehat{i \pi S_{1}^{z}}}\right)_{J_{1} M_{1} J_{1}^{\prime} M_{1}^{\prime}, J_{2} M_{2} J_{2}^{\prime} M_{2}^{\prime}} \\
& =\delta_{M_{1}-M_{2}, M_{1}^{\prime}-M_{2}^{\prime}} \sum_{\lambda \lambda^{\prime} m_{1} m_{2}} A_{J_{1} J_{2}}^{\lambda} A_{J_{1}^{\prime} J_{2}^{\prime}}^{\lambda^{\prime}}(-1)^{m_{1}} \\
& \times\left\langle 1 M_{1}-M_{2}, J_{2} M_{2} \mid J_{1} M_{1}\right\rangle\left\langle 1 M_{1}^{\prime}-M_{2}^{\prime}, J_{2}^{\prime} M_{2}^{\prime} \mid J_{1}^{\prime} M_{1}^{\prime}\right\rangle \\
& \left.\times\left\langle 1 m_{1} 1 m_{2} \mid \lambda M_{1}-M_{2}\right\rangle\left\langle 1 m_{1} 1 m_{2} \mid \lambda^{\prime} M_{1}-M_{2}\right\rangle\right\rangle
\end{aligned}
$$

We can actually set up $M_{1}=M_{1}^{\prime}$ and $M_{2}=M_{2}^{\prime}$ in (130) since in the computation of the string order parameter, the third component of the angular momenta is preserved. We have computed the highest eigenvalue $x_{\text {st }}$ of the matrix (130), which turns out to be smaller than one. This shows that $g(\ell)$ decays exponentially with a correlation length $\xi^{\text {st }}$ whose value is obtained by the eq.

$$
\xi^{\mathrm{st}}=-1 / \ln \left|x_{\mathrm{st}}\right|
$$

\section{FIGURE CAPTIONS}

Fig.1 Graphical representation of the MP ansatz (35) in the case of the spin 1/2 ladder and basis $|J M\rangle_{N}$ with $J=0$ and 1. Every dot represents a spin 1/2. A link between two dots denotes the formation of a singlet between the spins. Doted lines denote symetrization of the spins encircled by them.

Fig.2 The MP parameters for the ladder $A A_{1 / 2}$. In figures 2 to 10 we adopt the notation $x=\left|J_{\|} / J_{\perp}\right|$. The curve $A_{J_{1} J_{2}}^{\lambda}$ is labelled as $\left[J_{1}, J_{2}, \lambda\right]$.

Fig.3 Same notations as in fig. 2 but for the $F A_{1 / 2}$ ladder.

Fig.4 $J^{U}(A F) \equiv J_{\|}^{U}(A F)$ and $J^{U}(F A) \equiv J_{\|}^{U}(F A)$.

Fig.5 GS energy per site of the $A A$ and $A F$ ladders and their $U$ dual models.

Fig.6 Spin-correlation lengths of the $A A$ ladder and its $U$ dual.

Fig.7 GS energy per site of the $A F$ ladder and its $T$ dual given by the $F A$ ladder.

Fig.8 GS energy per site of the $A A$ ladder and its $S$ dual.

Fig.9 Spin-correlation length of the $A A$ ladder and its $S$ dual.

Fig.10 Plots of the spin-correlation length $\xi$ and the string correlation length $\xi^{\text {st }}$ of the ladder $A A_{1}$.

Fig.11 Pictorical representation of a possible AKLT state of the spin 1 ladder.

Fig.12 Graphical representation of a generic AKLT state of a ladder denoted as $(p, q, r)$. There are a total of $p+q+r$ dots inside every circle representing a total spin $S=(p+q+r) / 2$. 


$$
\begin{aligned}
& \mathbf{N}+\mathbf{1}=\mathbf{A}_{00}^{0} \quad \mathbf{N} \mathbf{\mathbf { O }}+\mathbf{A}_{01}^{1} \quad \mathbf{N} \\
& \mathbf{N}+19=A_{10}^{1} \quad \mathbf{N} \quad+A_{11}^{0} \quad \mathbf{N} \mathbf{O}^{\mathbf{0}}+\mathbf{A}_{11}^{1} \quad \mathbf{N}
\end{aligned}
$$

Fig. 1 
Fig. 2

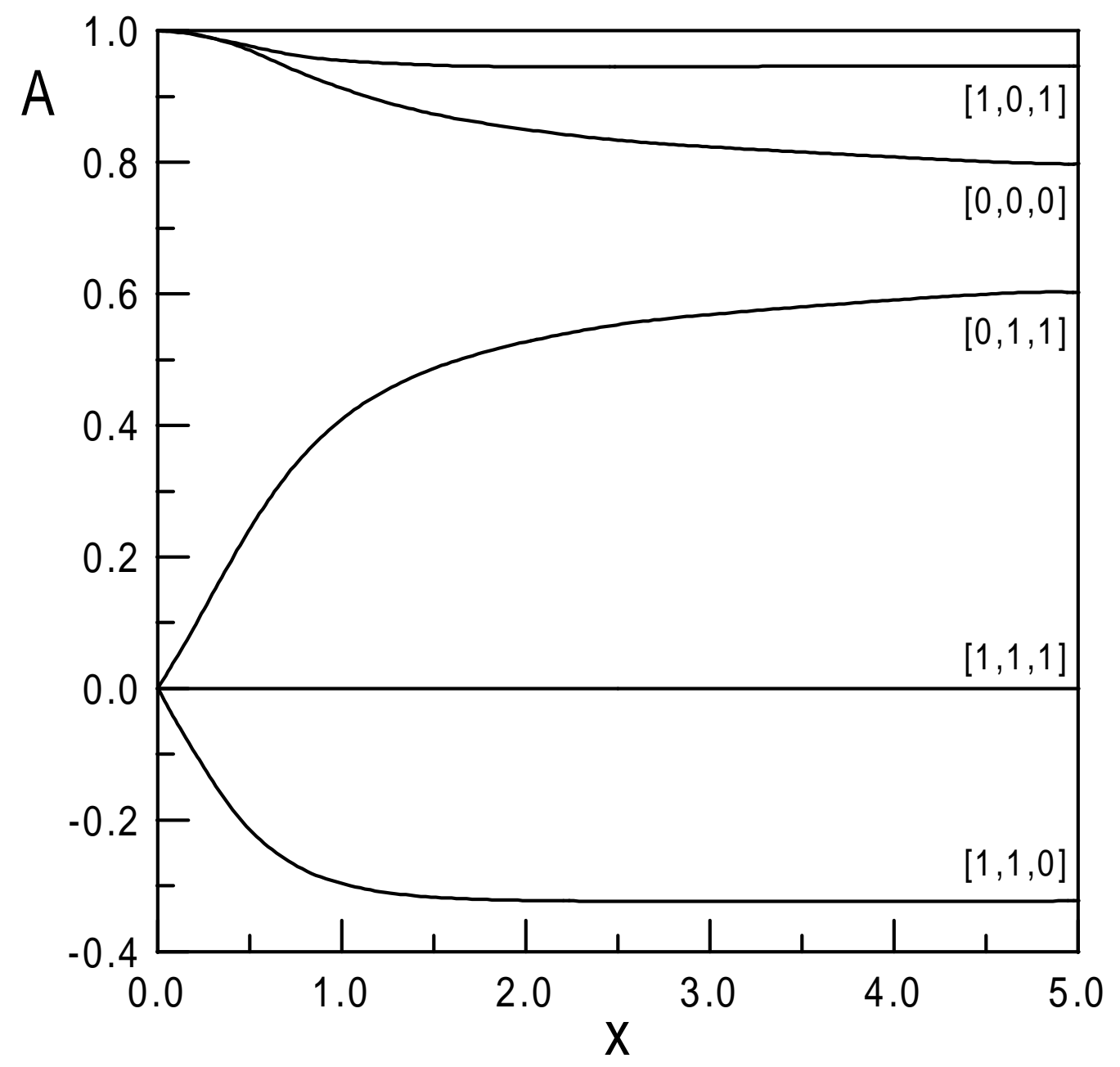


Fig. 3

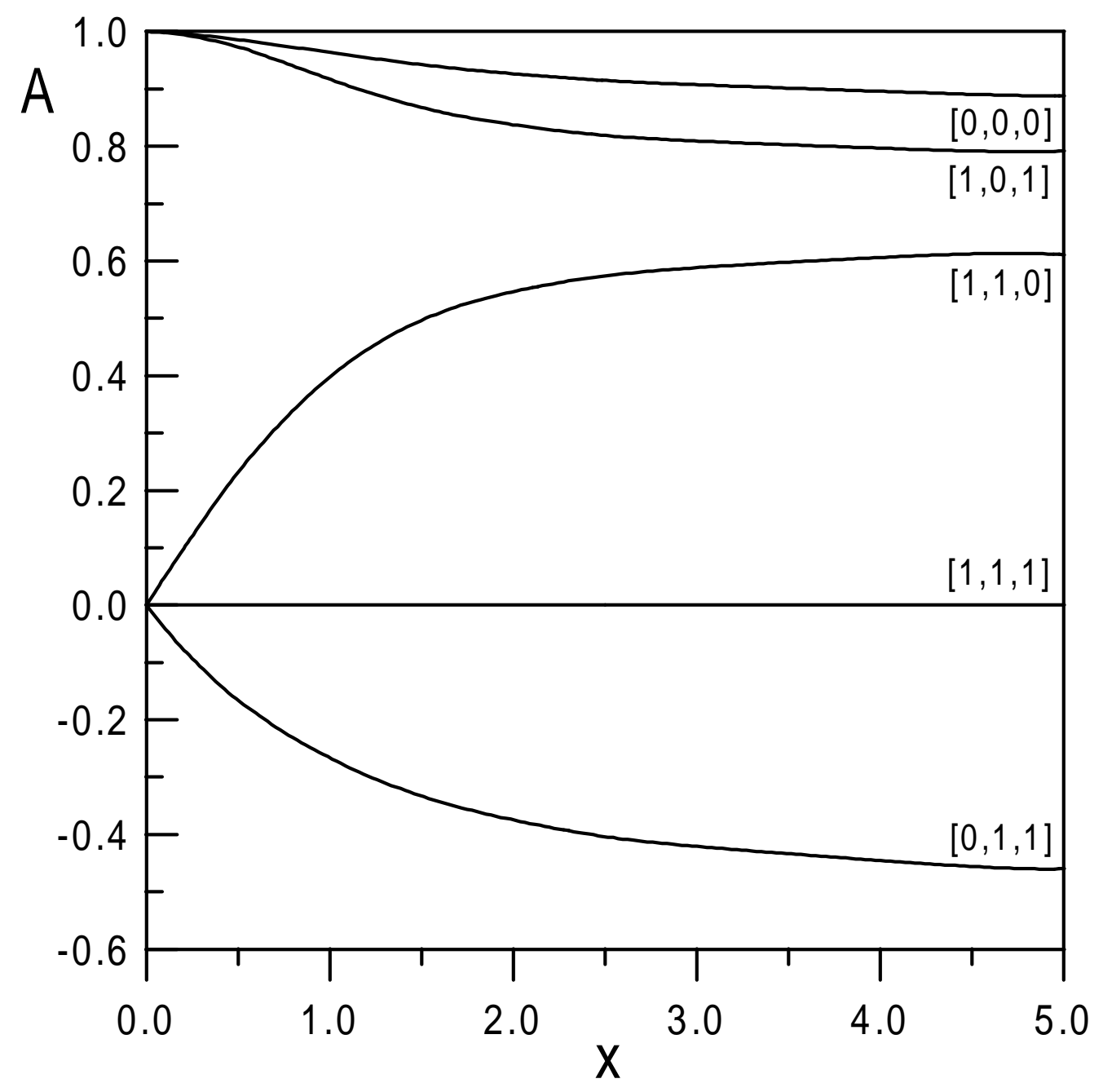


Fig. 4

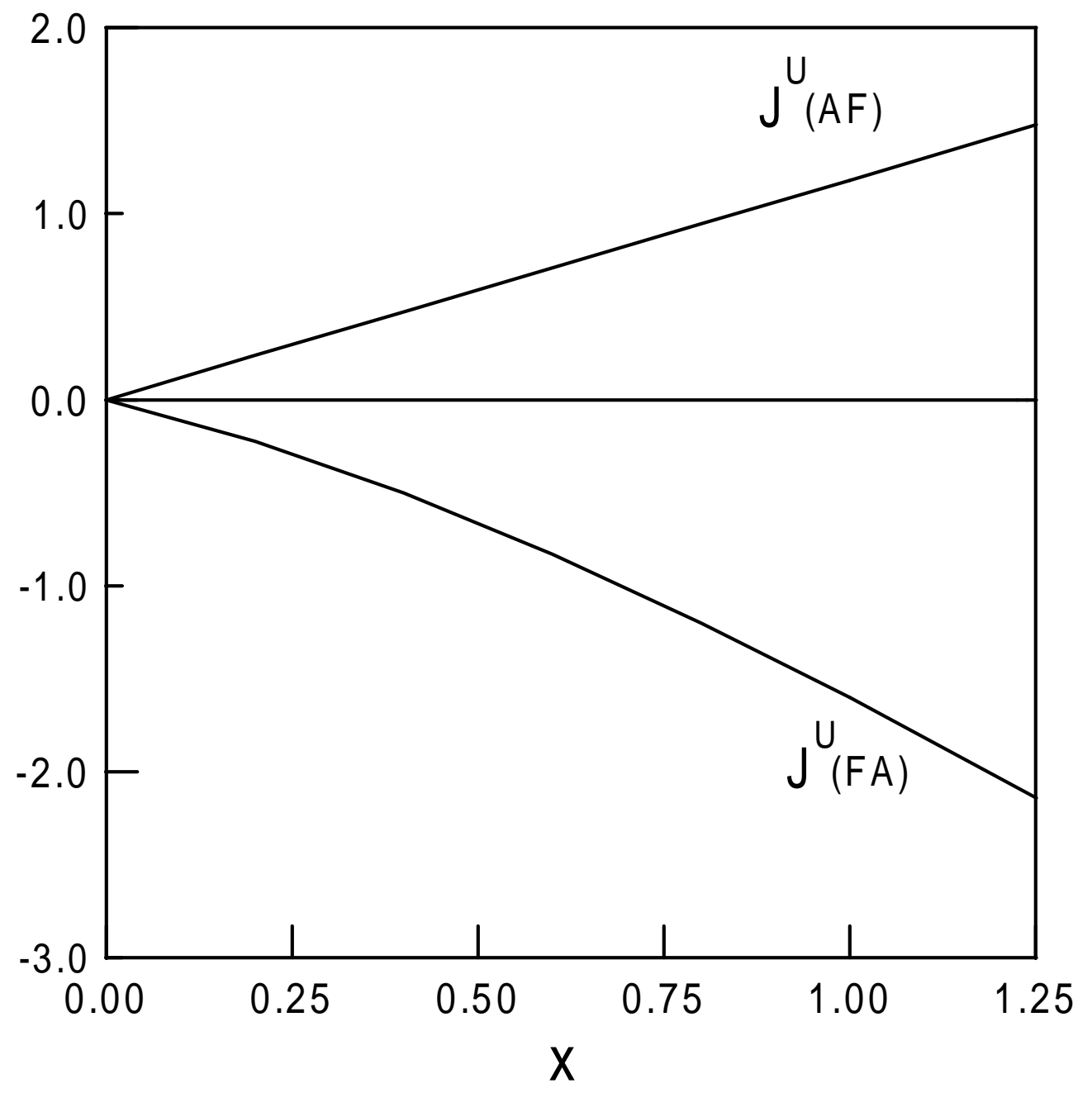


Fig. 5

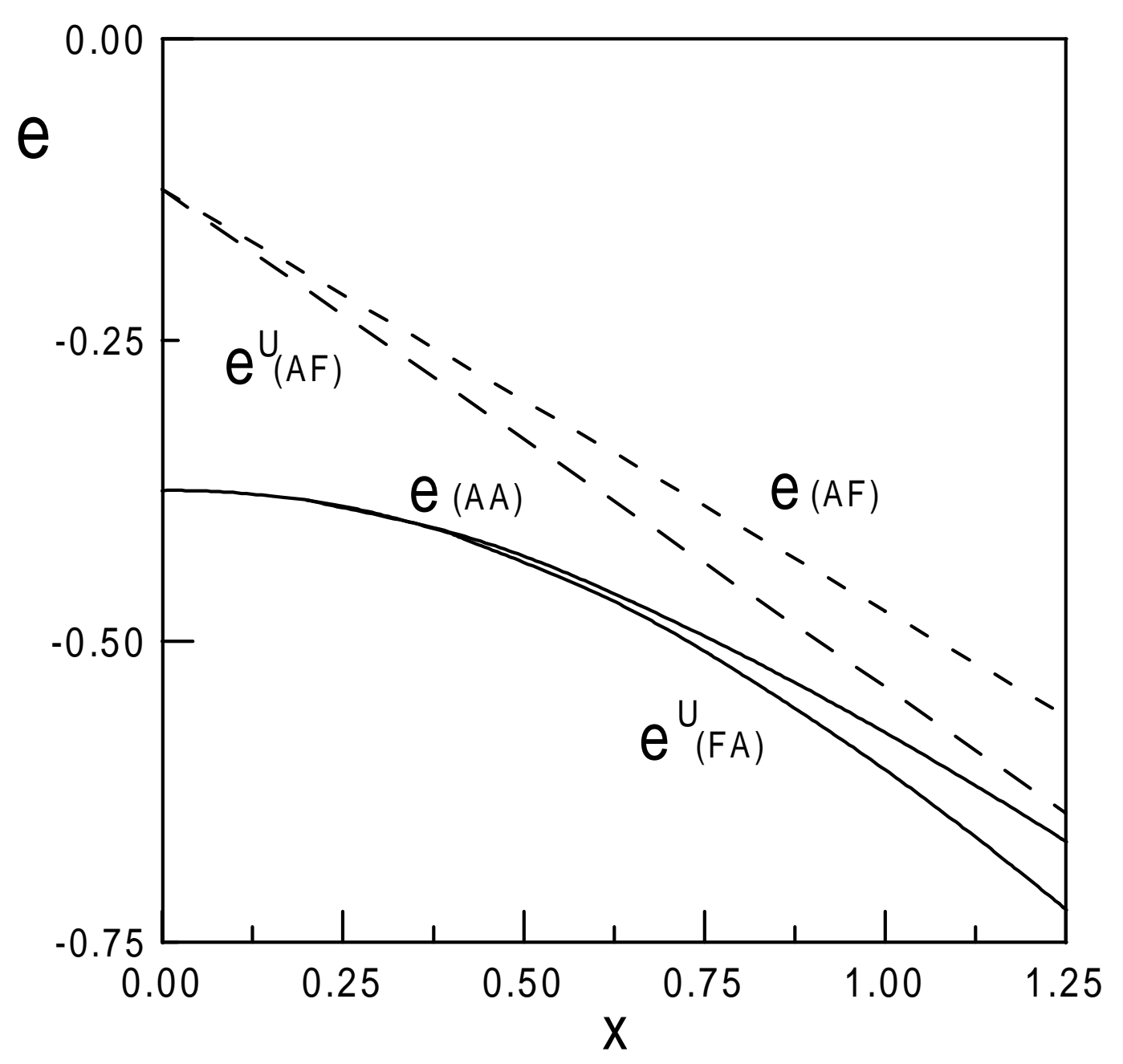


Fig. 6

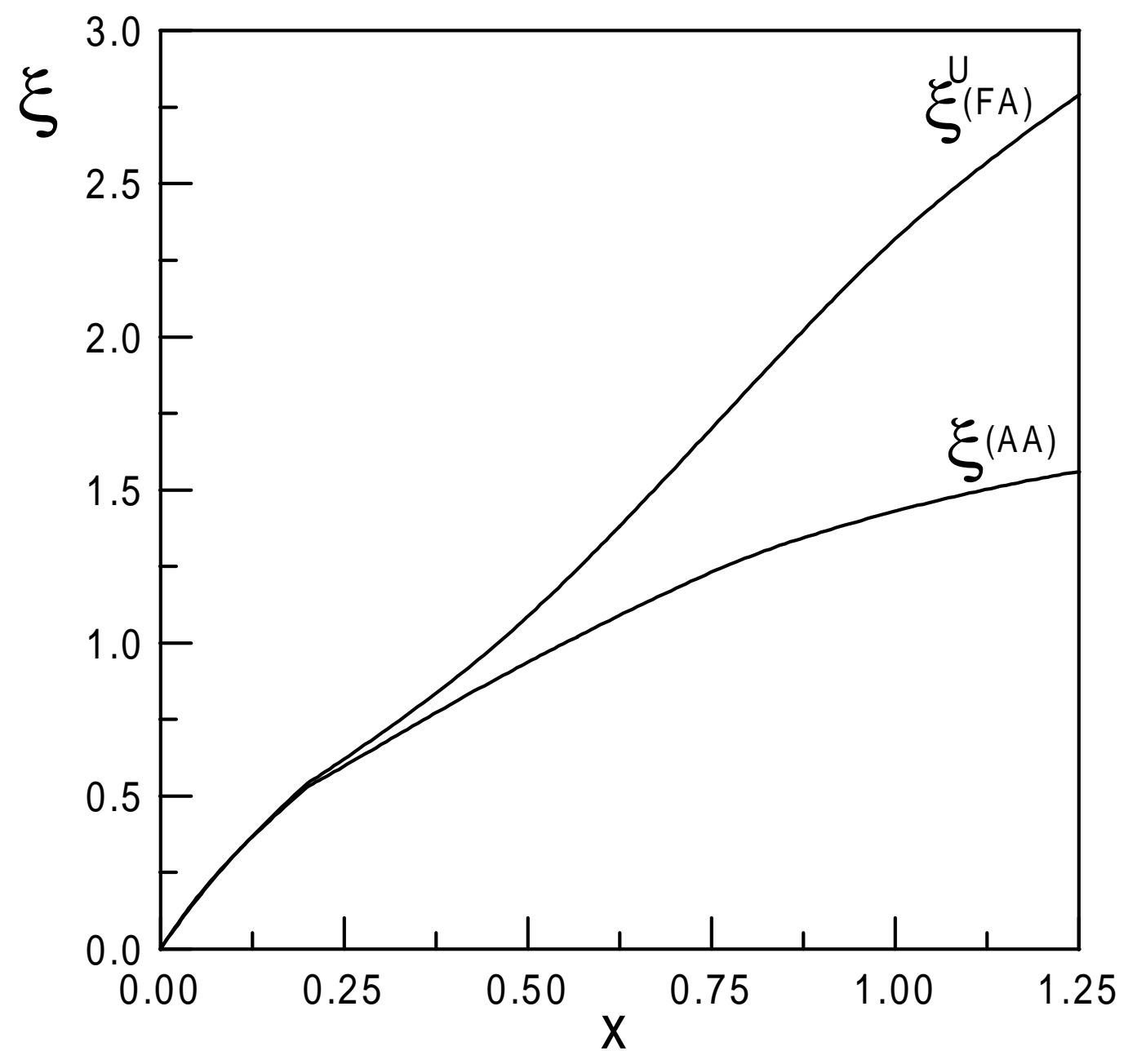


Fig. 7

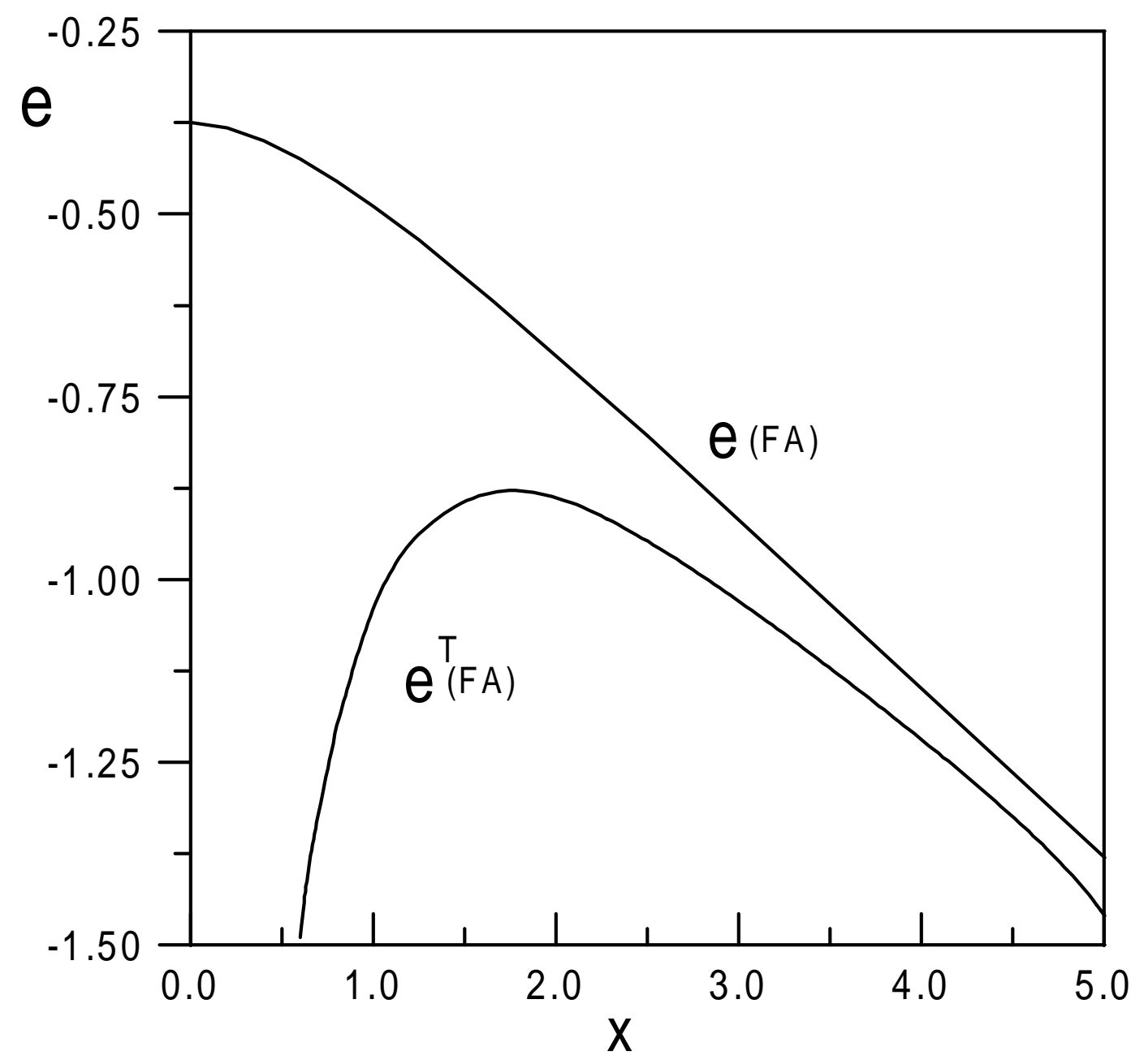


Fig. 8

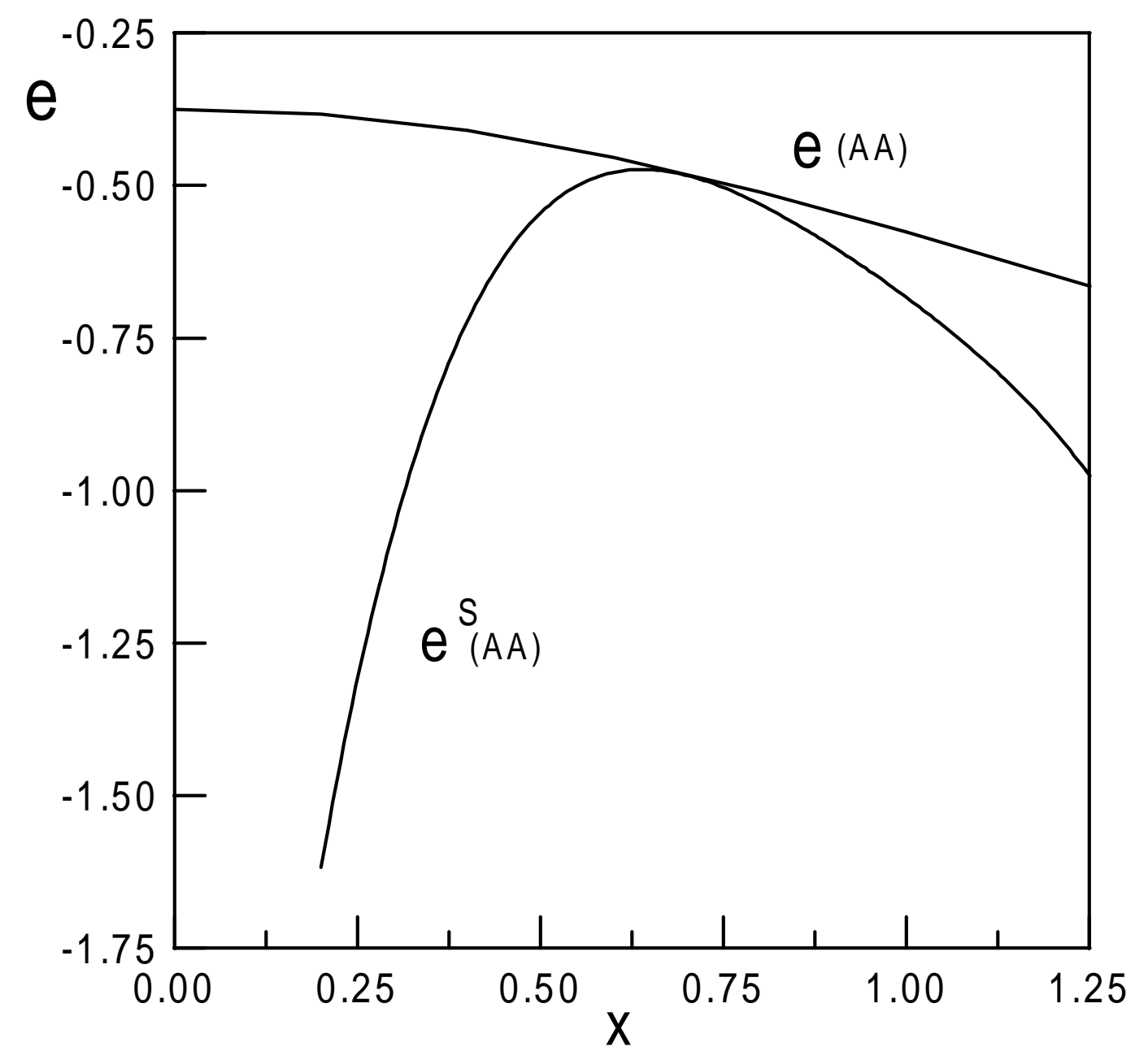


Fig. 9

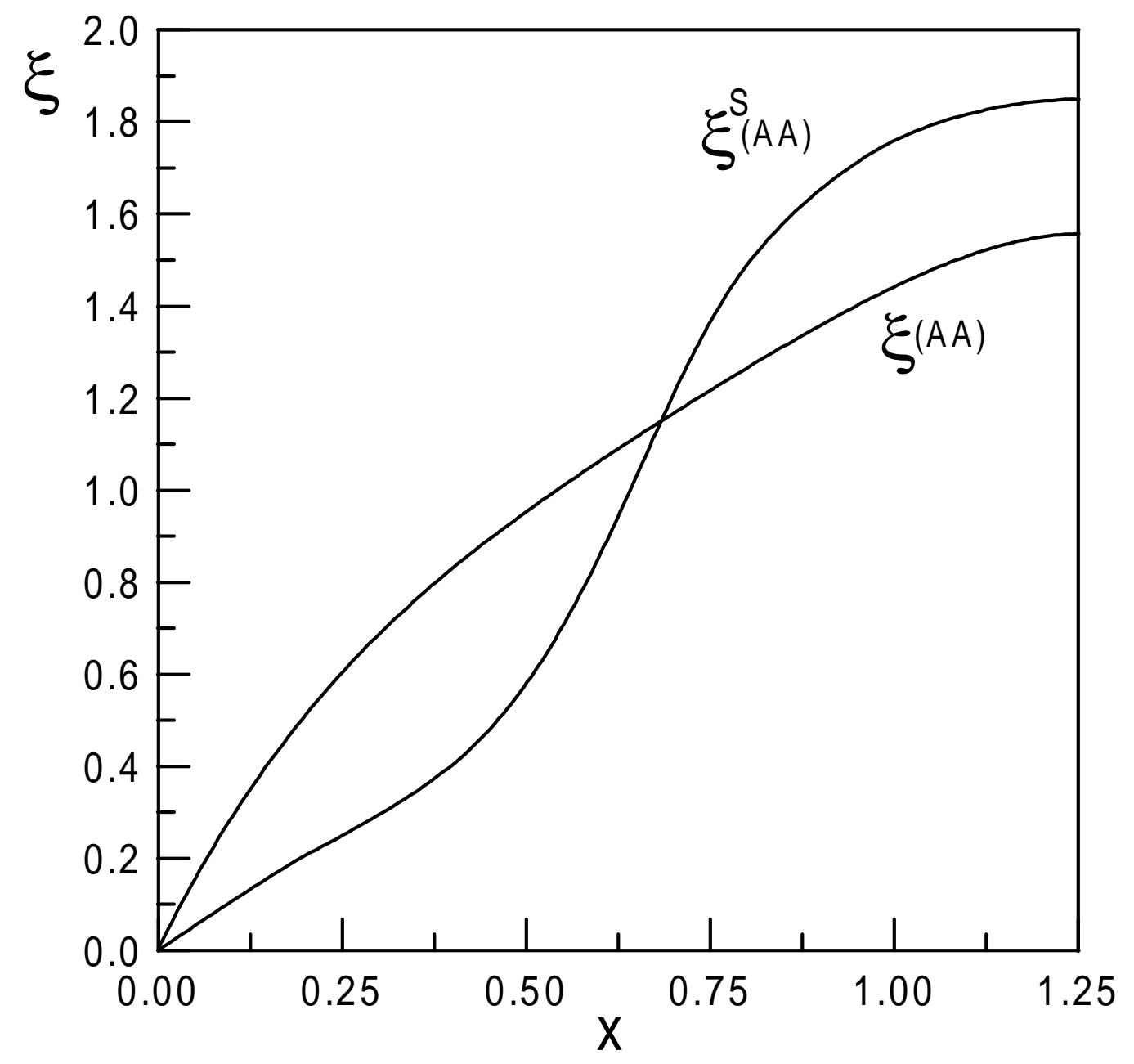


Fig. 10

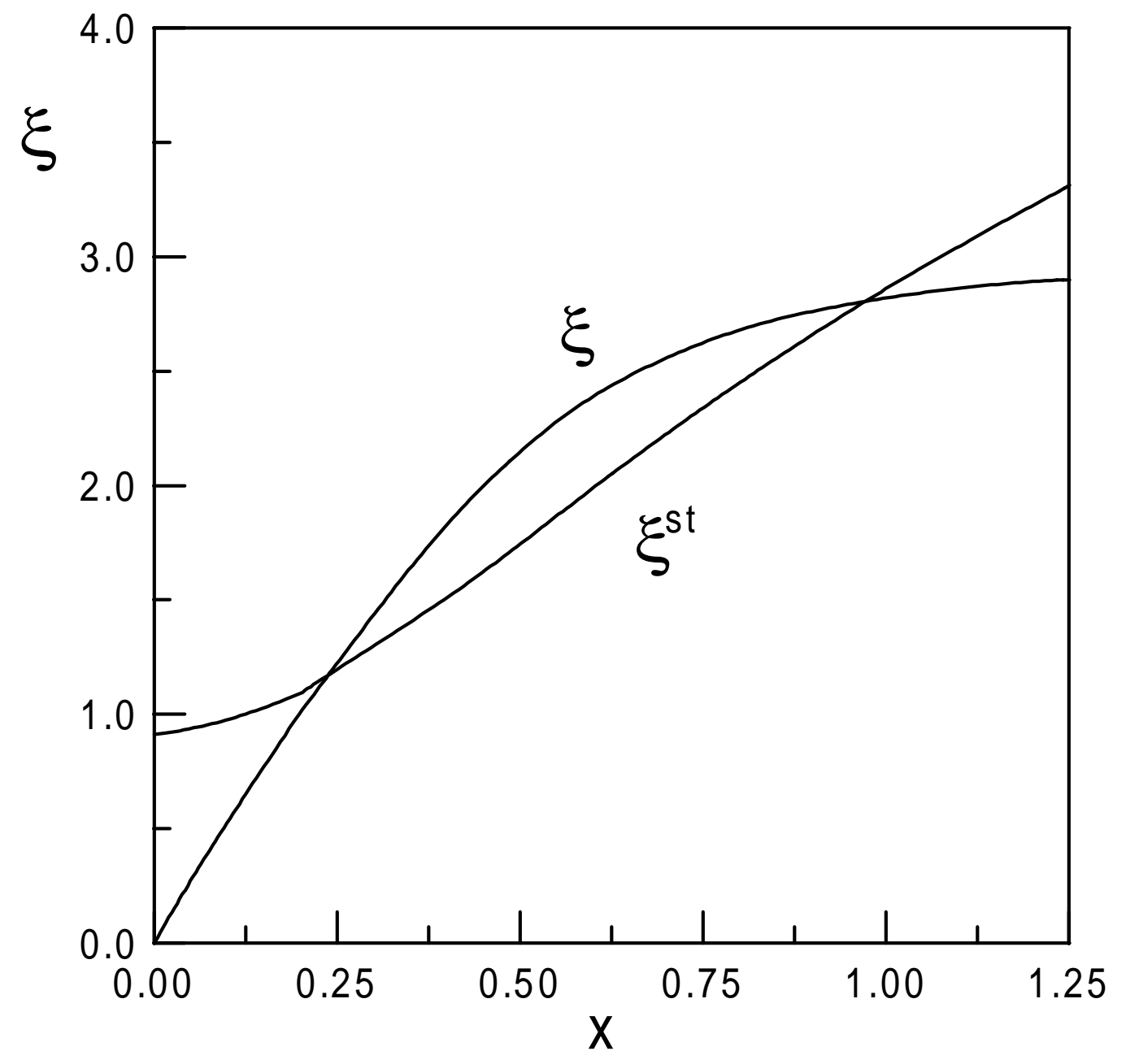



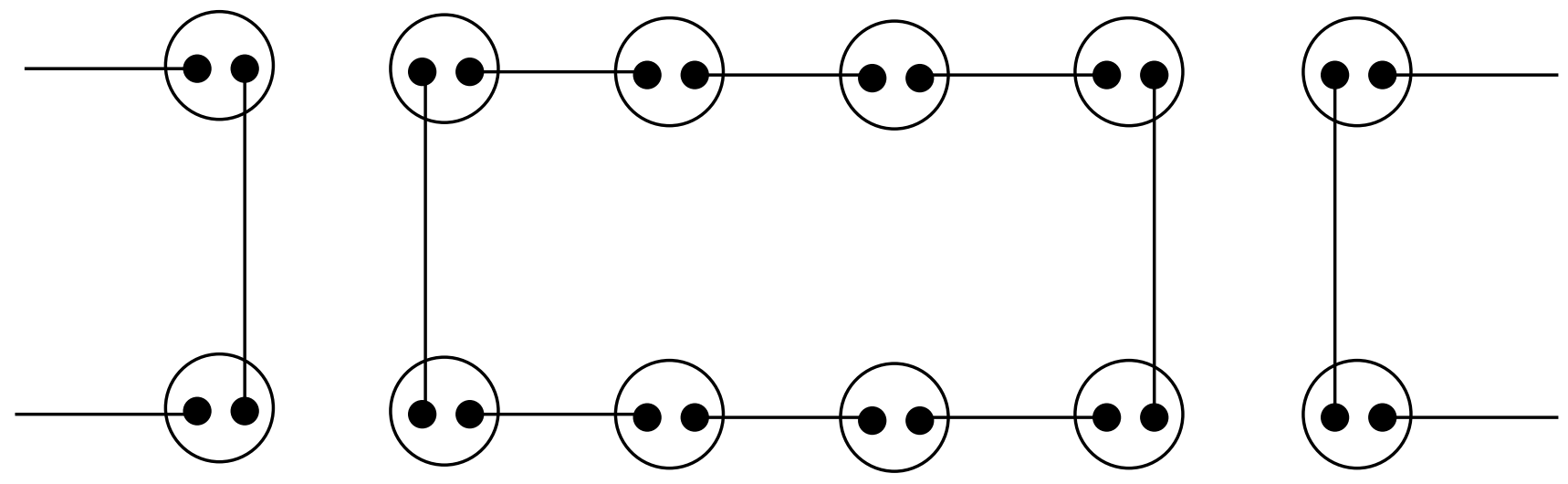

Fig. 11 


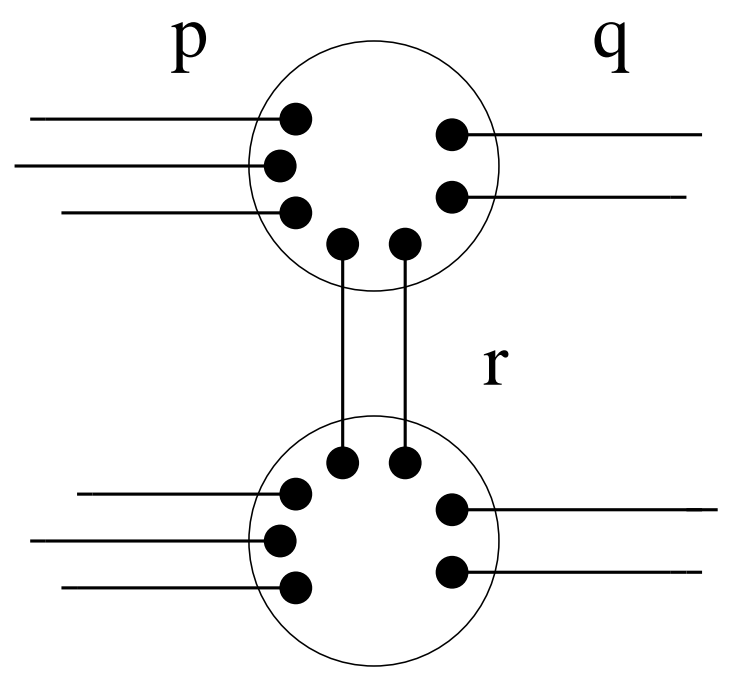

Fig. 12 